\title{
Efficient Energy Balancing Across Multiple Harmonics of Nonlinear Normal Modes
}

Dongxiao Hong ( $\nabla$ dx.hong@bristol.ac.uk)

University of Bristol Faculty of Engineering https://orcid.org/0000-0001-7861-8087

Thomas L. Hill

University of Bristol Faculty of Engineering

Simon A. Neild

University of Bristol Faculty of Engineering

\section{Research Article}

Keywords: Nonlinear normal modes (NNMs), Energy balancing analysis, Isolas, Semi-analytical study.

Posted Date: November 1st, 2021

DOI: https://doi.org/10.21203/rs.3.rs-1023425/v1

License: (1) This work is licensed under a Creative Commons Attribution 4.0 International License.

Read Full License 


\title{
Efficient Energy Balancing Across Multiple Harmonics of Nonlinear Normal Modes
}

\author{
Dongxiao Hong • Thomas L. Hill • Simon A. Neild
}

Received: date / Accepted: date

\begin{abstract}
Predicting the forced responses of nonlinear systems is a topic that attracts extensive studies. The energy balancing method considers the net energy transfer in and out of the system over one period, and establishes connections between forced responses and nonlinear normal modes (NNMs). In this paper, we consider the energy balancing across multiple harmonics of NNMs for predicting forced resonances. This technique is constructed by combining the energy balancing mechanism with restrictions (established via excitation scenarios) on external forcing and harmonic phase-shifts; a semi-analytical framework is derived to achieve both accurate/robust results and efficient computations. With known inputs from NNM solutions, the required forcing amplitudes to reach NNMs at resonances, along with their discrepancy, i.e. the harmonic phase-shifts, are computed via a one-step scheme. Several examples are presented for different excitation scenarios to demonstrate the applicability of this method, and to show its capability in accurately predicting the existence of an isola where multiple harmonics play a significant part in the response.
\end{abstract}

Keywords Nonlinear normal modes (NNMs), Energy balancing analysis, Isolas, Semi-analytical study.

Dongxiao Hong

Department of Mechanical Engineering, University of Bristol, Bristol, BS8 1TR, UK

E-mail: dx.hong@bristol.ac.uk

Thomas L. Hill

Department of Mechanical Engineering, University of Bristol, Bristol, BS8 1TR, UK

Simon A. Neild

Department of Mechanical Engineering, University of Bristol, Bristol, BS8 1TR, UK

\section{Introduction}

Close to the boundaries of their performance envelope, many engineering systems can experience nonlinear phenomena $[21,6,40]$. For example, an aircraft may be designed to be highly flexible to gain aerodynamic efficiency which, in turn, induces significant nonlinear behaviours during operation [34]; micro-electromechanical systems can be employed as innovative bandpass filters with improved performance by exploiting the modal coupling due to nonlinearity [7].

To understand these nonlinear behaviours, the extension of linear modal analysis to account for nonlinearity has been extensively considered. An early approach was proposed by Rosenberg [29,30], where a nonlinear normal mode (NNM) is defined as a synchronous periodic response of a undamped and unforced, or conservative, system. This definition was later relaxed to a periodic response (not necessarily synchronous) of a conservative system [18]. Alternative definitions of a nonlinear mode include a damped NNM $[33,37]$, a spectral submanifold (SSM) [8], and a phase resonance nonlinear mode [39]. Using the concept of nonlinear modes, many nonlinear behaviours have been investigated, e.g. modal interactions, bifurcations and instability $[1,38,19,13,15,14]$, to facilitate the understanding of their mechanisms and aid practical design. In addition, owing to their invariance property, analysis of large-scale nonlinear systems also becomes feasible via model order reduction techniques [17,38, 8, 22].

In practice, engineering systems are usually operating under forced conditions, where the forced response curves (FRCs) are widely considered for analysis and design of nonlinear systems $[18,40]$. Employing an energy-based framework, the forced responses can be used to extract NNMs for modal analysis [23]; or vice 
versa - using NNMs to interpret the forced responses [12].

In [23], a nonlinear extension of the force appropriation technique was developed in order to identify NNMs in experimental setups. To this end, a set of multipoint multi-harmonic excitations are tuned to exhibit phase quadrature with all harmonics of displacements, in order for the excitations to compensate the damping effect. Successful applications of the force appropriation technique were demonstrated in a large number of studies in combinations with, for example, resonant decay [24], control-based continuation [27,26] and phase-locked loop $[25,4]$. These studies show that, for most cases, even a single excitation is sufficient to accurately locate the NNM branches. However, numerical and experimental studies also show limitations and significant errors, arising from the insufficient compensation between the single-location forcing and distributed damping of the structure [28]. Alternative approaches, e.g. response-controlled stepped-sine testing [16] and velocity feedback [31], can as well be utilised for nonlinear modal testing.

For nonlinear systems operating under forced conditions, the resonances are of particular interest as they usually represent the most significant responses in a system. Near resonances, the forced responses may be seen as perturbations from NNMs $[12,2]$. Using these observations, an energy-balancing method was used to establish the relationships between forced responses and NNM branches [12]. This is achieved by assuming resonances as phase-shifts of NNMs and considering the energy balancing during periodic responses - the net energy transfer in and out of any mode must be zero. The energy balancing method has also been used to predict the presence of isolas $[19,9,5]$ - i.e. regions of forced responses that are separated from the primary branches of the response; to quantify the relative importance of NNMs by predicting the forcing amplitude required to reach them at resonance [20]; and to identify an appropriate number of excitations and their locations in conducting force appropriation tests [28]. This energy balancing method was originally derived based on systems with conservative nonlinearity and, recently it was extended to account for non-conservative nonlinearity with applications in full-scale structures [35]. This extended energy balancing method has been compared with nonlinear modal synthesis in predicting resonances [36].

To complicate matters, not all NNMs are pertinent to forced responses - only those NNMs whose fundamental components are coupled with specific phase relationships correspond to forced responses when forcing and damping are applied $[10,11]$. In $[2]$, when a conser- vative NNM may be perturbed to forced responses are quantified via Melnikov analysis. Another limitation of the energy balancing technique, proposed in [12], lies in the assumption that using a single harmonic is representative of the modal response. This single-harmonic assumption may fail to capture resonances where multiple harmonics are involved. Such a limitation was demonstrated in [5], where significant errors, partly arising from the single harmonic assumption, can be observed during the emergence of an isola to the primary forced response curve.

Being a versatile technique with applications in both theoretical and experimental studies, one of the main advantages of the energy balancing method is its analytical framework, which in turn can bring inaccuracy in complex application scenarios as it is typically assumed that only the fundamental harmonic is significant. However, the analytical framework does not allow for a direct extension to account for multiple harmonics, instead, computationally expensive numerical schemes are required. In this study, we present an extended technique that overcomes the single-harmonic limitations, whilst preserving the computational efficiency by employing a semi-analytical scheme. To this end, the rest of the paper is organised as follows.

Firstly in $\S 2$, the mechanism of energy balancing during periodic responses, proposed previously in the literature, is reviewed highlighting the relationships between forced responses and NNMs. A nonlinear beam system is considered to show to applicability and limitations of single-harmonic energy balancing method. The single-harmonic energy balancing method is then extended to account for multiple harmonics in $\S 3$, when quadrature excitations are applied to the modal harmonics. A semi-analytical framework is obtained by combining energy balancing across harmonics and forcing/phase-shift constraints. In $\S 4$, this technique is further extended to more practical scenarios where the excitations are in quadrature with physical displacements. Throughout the paper, the application of the proposed method is compared with the force appropriation technique [23] and single-harmonic energy balancing method [12] via a series of examples.

\section{Energy-transfer balancing in nonlinear systems: from system to harmonic levels}

This section first reviews the mechanism of energy balancing of nonlinear systems during periodic responses. This is achieved by demonstrating the energy-transfer 
balancing at the level of the system and of the mode $e^{1}$, and then to that of the harmonic. From such an energybased perspective, the relationships between nonlinear normal modes (NNMs) and forced responses are highlighted. Discussions in this section provide a basic concept which we build on in this paper, where an efficient technique for applying Harmonic-level Energy Transfer Analysis (HETA) is proposed - as introduced in $\S 3$ and $\S 4$.

\subsection{Energy-transfer balancing at the system level}

For a nonlinear system, its dynamics can be expressed, using its modal components, in the form

$\ddot{\mathbf{q}}+\mathbf{D} \dot{\mathbf{q}}+\mathbf{\Lambda} \mathbf{q}+\mathbf{N}_{q}(\mathbf{q})=\mathbf{p}(t)$,

where $\ddot{\mathbf{q}}, \dot{\mathbf{q}}$ and $\mathbf{q}$ are vectors of modal accelerations, velocities and displacements respectively. The diagonal matrix $\mathbf{D}$ contains the linear damping coefficients, i.e. it is assumed that the modal damping is decoupled, and $\boldsymbol{\Lambda}$ is a diagonal matrix containing the squares of the linear natural frequencies. Vector $\mathbf{N}_{q}(\mathbf{q})$ contains the nonlinear stiffness terms (assumed to be conservative, and a function of the displacements, q). The external periodic forcing is captured by the vector $\mathbf{p}$.

At the system level, energy-transfer balancing considers the net energy transfer in and out of a system over one period of motion. As the nonlinear terms considered here are conservative, this energy transfer can only be achieved via the damping and external forcing terms. Over one period, the total energy loss by the system due to these nonconservative terms must sum to zero, such that

$E_{D}+E_{P}=0$,

where $E_{D}$ and $E_{P}$ denote the damping and forcing energy-transfer terms respectively.

In the modal domain, the nonlinear system, described by Eq. (1), can be seen as a collection of modes, where the equation of motion of the $i^{\text {th }}$ mode is written

$\ddot{q}_{i}+d_{i} \dot{q}_{i}+\omega_{n i}^{2} q_{i}+N_{q i}(\mathbf{q})=p_{i}(t)$,

and where $d_{i}, \omega_{n i}$ and $p_{i}$ are the $i^{\text {th }}$ modal damping coefficient, linear natural frequency and external modal forcing respectively. As such, the energy-transfer terms in Eq. (2) can be translated, using modal components, into summation form

$\sum_{i=1}^{N}\left(E_{D i}+E_{P i}\right)=0$,

1 Note that the term mode is used to refer to a mode of the underlying linear model of the system. (a) - On an NNM

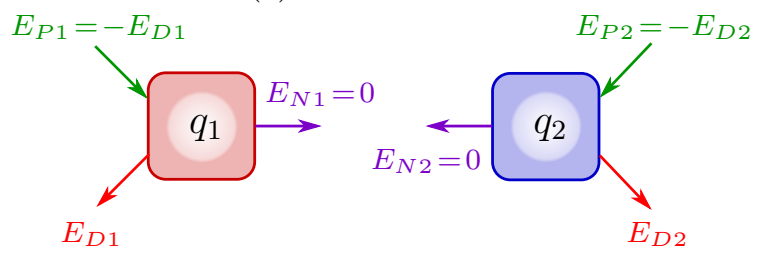

(b) - Not on an NNM

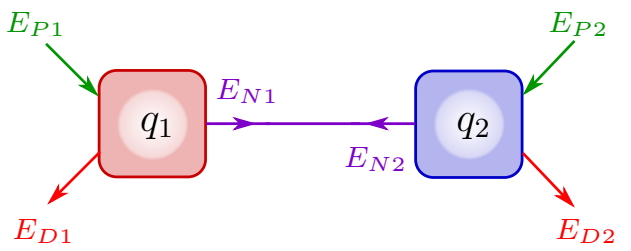

Fig. 1 A schematic of the energy transfer at the mode level, for a two-mode system. Panel $(a)$ shows the case where the forcing and damping match precisely, so that the response may equal an NNM. In panel (b) the forcing and damping do not match and the response is not precisely on the NNM.

where $E_{D i}$ and $E_{P i}$ are the $i^{\text {th }}$ modal damping and forcing energy-transfer terms respectively, and $N$ is the total number of modes in the system. Note that, the nonlinearities, described by $N_{q i}(\mathbf{q})$, may lead to coupling and energy transfer between modes, where the net energy tranfer to the $i^{\text {th }}$ mode is defined as $E_{N i}$. The effect of $E_{N i}$ can be seen as internal energy rearrangement among modes; hence, it exhibits a conservative effect at the system level and has zero contribution to the energy balancing in Eqs. (2) and (4).

\subsection{Energy-transfer balancing at the mode level}

The concept of energy balancing analysis can be extended to the mode level by considering the energy balancing for each modal component. At this level, besides damping and forcing energy-transfer terms, the nonlinearity-induced energy transfer between modes, $E_{N i}$, is seen as an external energy source, and needed to be accounted for. For a periodic response, the net energy transfer in and out of any mode over one period must be zero, i.e.

$E_{D i}+E_{P i}+E_{N i}=0$.

This describes the energy balancing at the mode level, similar to Eq. (2) for that at the system level.

At the mode level, for an NNM (i.e. undamped and unforced periodic response), the net energy transfer between modes must be zero, i.e. $E_{N i}=0$, as no mode may lose or gain energy over one period and still remain periodic. Therefore, if an NNM solution is precisely equal to a forced response, Eq. (5) implies that 


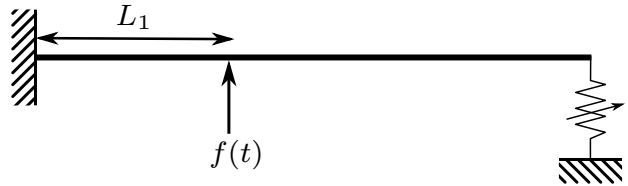

Fig. 2 A schematic of a cantilever beam with a nonlinear spring at the free end.

the forcing energy gain must be precisely equal to the damping energy loss for each mode, i.e.

$E_{P i}=-E_{D i}$.

A schematic of this energy transfer is shown in Fig. 1(a), for a two-mode system.

In most applications, however, the applied forcing cannot precisely satisfy Eq. (6), i.e. the forcing energy gain for each mode does not equal the damping energy loss (for example, a mode may be damped but unforced). Such a forcing case is referred to here as an imperfect forcing. Therefore, Eq. (5) reveals that, for an imperfect forcing, $E_{N i} \neq 0$, which violates a condition of an NNM (i.e. that $E_{N i}=0$ ). A schematic of this energy transfer is shown in Fig. 1(b). This implies that an NNM can never be precisely reached by a system with imperfect forcing. To quantify the deviation in the energy-transfer from Fig. 1( $a$ ) to Fig. 1(b), caused by imperfect forcing, the effect of non-zero nonlinear energy-transfer terms, $E_{N i}$, may be considered.

In Ref. [12], the forced response in the neighbourhood of resonance, where $E_{N i} \neq 0$, is considered as a perturbation from an NNM. In order for a system to exhibit an internal energy transfer, $E_{N i}$, a perturbation in the phase of the modal components (called a phaseshift) is required. If this phase-shift is sufficiently small, it may be assumed that the forced response is close to that of an NNM; conversely, a large phase-shift corresponds to a large change in the response, indicating that the forced response is significantly different to the NNM. Note that, this technique assumes the dynamics of a mode may be approximated by a single harmonic, and is established using Eqs. (5) and (6), hence it is termed the Mode-level Energy Transfer Analysis (META) in this paper.

To demonstrate the application of META, a motivating example is considered, shown in Fig. 2 - a cantilever beam with a cubic nonlinear spring at the free end, and excited at a point on the part-span position, $L_{1}$, by a single-harmonic force

$f(t)=F_{1} \cos (\Omega t)$.

The deflection of this beam is modelled using the first two modes, and the modal equations of motion are given by

$\ddot{q}_{1}+d_{1} \dot{q}_{1}+\omega_{n 1}^{2} q_{1}+N_{q 1}\left(q_{1}, q_{2}\right)=\theta_{1} f(t)$, $\ddot{q}_{2}+d_{2} \dot{q}_{2}+\omega_{n 2}^{2} q_{2}+N_{q^{2}}\left(q_{1}, q_{2}\right)=\theta_{2} f(t)$,

where $\theta_{i}$ is the modeshape of the $i^{\text {th }}$ mode at the excitation point, and where $d_{i}=2 \omega_{n i} \zeta$ where $\zeta$ is the modal damping. The nonlinear terms are given by

$N_{q 1}=\alpha_{1} q_{1}^{3}+3 \alpha_{2} q_{1}^{2} q_{2}+\alpha_{3} q_{1} q_{2}^{2}+\alpha_{4} q_{2}^{3}$,

$N_{q 2}=\alpha_{2} q_{1}^{3}+\alpha_{3} q_{1}^{2} q_{2}+3 \alpha_{4} q_{1} q_{2}^{2}+\alpha_{5} q_{2}^{3}$,

where $\alpha_{i}$ denote nonlinear modal coefficients. The linear stiffness of the spring is tuned such that the two modes exhibit a 1:3 response, i.e. the fundamental (largest) harmonic of the second mode responds at three times the frequency of the fundamental harmonic of the first mode. As this system (which is analogous to that considered in [32]) is used as an illustrative example, a derivation of the expressions is not provided here; however, interested readers are directed to Refs. [32,40] for an overview of the approach taken for this derivation.

Here an example system with parameters given in Table 1 is considered. The first NNM branch (i.e. the locus of NNMs emerging from the first linear natural frequency) of the beam is found using the numerical continuation software COCO [3]. It is shown as a blue line in Fig. 3(a)(ii) in the projection of the response frequency, $\omega$, against the maximum response amplitude of the second mode, $Q_{2}$.

A periodic force, $f(t)$, is applied at a part-span position, $L_{1}=0.7 \mathrm{~L}$, to consider forced responses of the beam. The relationship between the NNM branch and the forced responses is identified via META using Eq. (6), and shown in Fig. 3(a)(i) - the forcing amplitude, $F_{1}$, required for the forced resonant responses to share solutions with the NNM branch at response frequency, $\omega$. Considering a forcing amplitude of $F_{1}=0.0275$, denoted as the dotted line in panel (a) $(i)$, META predicts three intersections (indications of three resonances), and they are labelled as dots in both panels. To verify this prediction, the corresponding forced responses are found using numerical continuation and shown as red lines in panel $(a)(i i)$. Excellent agreements are achieved for both the primary and the isolated forced responses; hence the prediction well captures the resonant responses.

The excitation location is then moved from $L_{1}=$ $0.7 \mathrm{~L}$ to $L_{1}=0.2 \mathrm{~L}$, and the energy balancing relationship is again computed via META, and shown in Fig. 3(b) $(i)$. Considering a forcing amplutude of $F_{1}=$ 0.18 , i.e. the dotted line, META predicts one intersection (the dot) between the forced responses and the NNM branch. In comparison, the responses for this forcing amplitude are computed numerically and shown as red lines in panel $(b)(i i)$. Results obtained via META capture the resonance on the primary forced responses the dot is in line with the primary resonant peak; whilst, 
Table 1 The parameters of the two-mode beam model, described by Eqs. (8) and (9).

\begin{tabular}{r|cc|cc|ccccc}
\hline Parameter & $\omega_{n 1}$ & $\omega_{n 2}$ & $d_{1}$ & $d_{2}$ & $\alpha_{1}$ & $\alpha_{2}$ & $\begin{array}{c}\alpha_{3} \\
\left(\times 10^{7}\right)\end{array}$ & $\alpha_{4}$ & $\alpha_{5}$ \\
\hline Value & 43.35 & 134.1 & 0.434 & 1.341 & 3.267 & -4.005 & 14.73 & -6.017 & 7.376
\end{tabular}

(a)

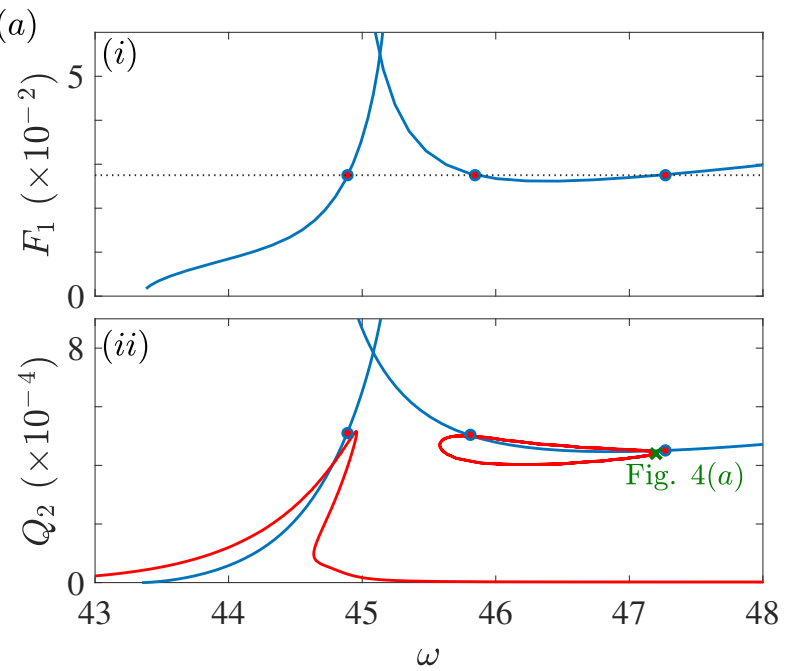

(b)

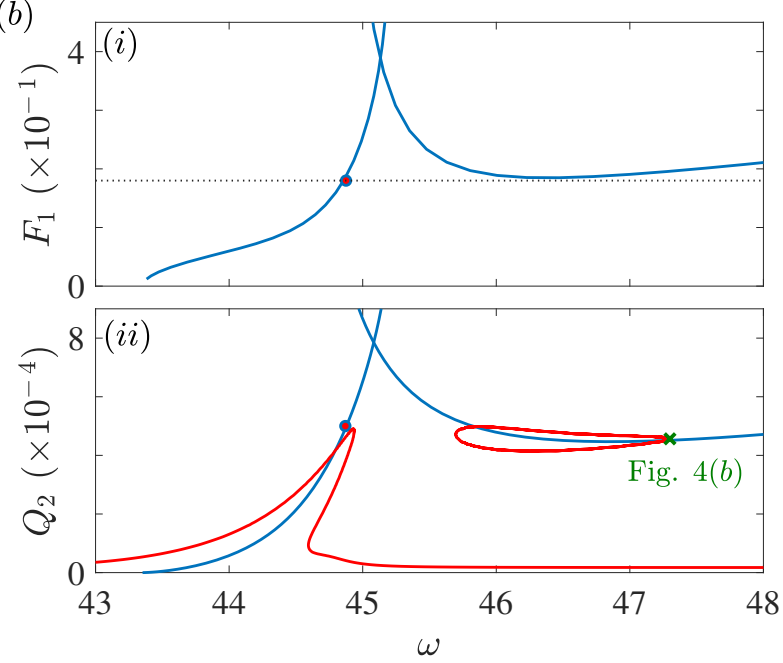

Fig. 3 Energy balancing analysis via Mode-level Energy Transfer Analysis (META). (a) Predicting resonances when the excitation is applied at $L_{1}=0.7 \mathrm{~L}$. Panel $(i)$ shows the forcing amplitude, $F_{1}$, required to share solutions with the first NNM branch at frequency $\omega$. Panel (ii) shows the first NNM branch (blue line) and the forced responses (red lines) for the two-mode beam in the projection of the response frequency, $\omega$, against the maximum displacement amplitude of the second linear mode, $Q_{2}$. The identified relationships between the NNM branch and the forced responses for an applied forcing $F_{1}=0.0275$, are labelled by dots in both panels. The periodic energy transfer on the forced response, labelled with a cross, is shown in Fig. 4(a). (b) Predicting results when the excitation is applied at $L_{1}=0.2 L$. The indentified relationships between the NNM branch and the forced responses for an applied forcing $F_{1}=0.18$, are labelled by dots in both panels. The periodic energy transfer on the forced response, labelled with a cross, is shown in Fig. 4(b).

unlike the case shown in Fig. 3(a), META fails to capture the resonances on the isolated forced responses, as no intersections are predicted.

To explain why META succeeds for the case where the forcing location is at $L_{1}=0.7 L$, but fails when $L_{1}=0.2 L$, the energy balancing at the mode level, described by Eq. (5), is further extended to consider the periodic energy transfer between harmonics. As with the interpretation that a system may be seen as a collection of modes, a mode may be seen as a collection of harmonics. This allows the modal coordinates to be expressed as a sum of harmonics

$q_{i}=\sum_{j \in \mathcal{H}_{i}} u_{i, j}$

where $u_{i, j}$ is the $j^{\text {th }}$ harmonic of the $i^{\text {th }}$ mode, and $\mathcal{H}_{i}$ is a set denoting the harmonics in the $i^{\text {th }}$ mode. Considering Eq. (10), the energy transfer at the mode level, Eq. (5), can be expressed in summation form

$\sum_{j \in \mathcal{H}_{i}}\left(E_{D i, j}+E_{P i, j}+E_{N i, j}\right)=0$

where the terms within the summation represent the net energy transfer to the $j^{\text {th }}$ harmonic of the $i^{\text {th }}$ mode due to the damping, forcing and nonlinear terms, respectively, and they may be computed using

$$
\begin{aligned}
& E_{D i, j}=\int_{0}^{T}\left[d_{i} \dot{q}_{i}\right] \dot{u}_{i, j} \mathrm{~d} t, \\
& E_{P i, j}=-\int_{0}^{T}\left[p_{i}(t)\right] \dot{u}_{i, j} \mathrm{~d} t, \\
& E_{N i, j}=\int_{0}^{T}\left[N_{q i}(\mathbf{q})\right] \dot{u}_{i, j} \mathrm{~d} t .
\end{aligned}
$$

Here, the periodic energy transfer at resonances on the isolas, i.e. the ' $x$ ' signs in Fig. $3(a)$ and $(b)$, are considered (resonances where META succeeds and fails in predictions respectively). Using Eqs. (12), the periodic energy-transfer terms may be computed for each harmonic, and they are shown in Figs. 4(a) and 4(b) with repect to cases in Fig. $3(a)$ and $(b)$.

Applying an excitation with forcing frequency, $\Omega$, near the first natural frequency gives energy to the fundamental component of the first mode, $u_{1,1}$, and the first harmonic of the second mode, $u_{2,1}$. When the forcing is at $L_{1}=0.7 \mathrm{~L}$, Fig. $4(a)$ shows that the major energy input is directed to $u_{1,1}$, with only one percent of energy imparted to $u_{2,1}$. Whilst the major internal energy transfer, triggered by nonlinear coupling 

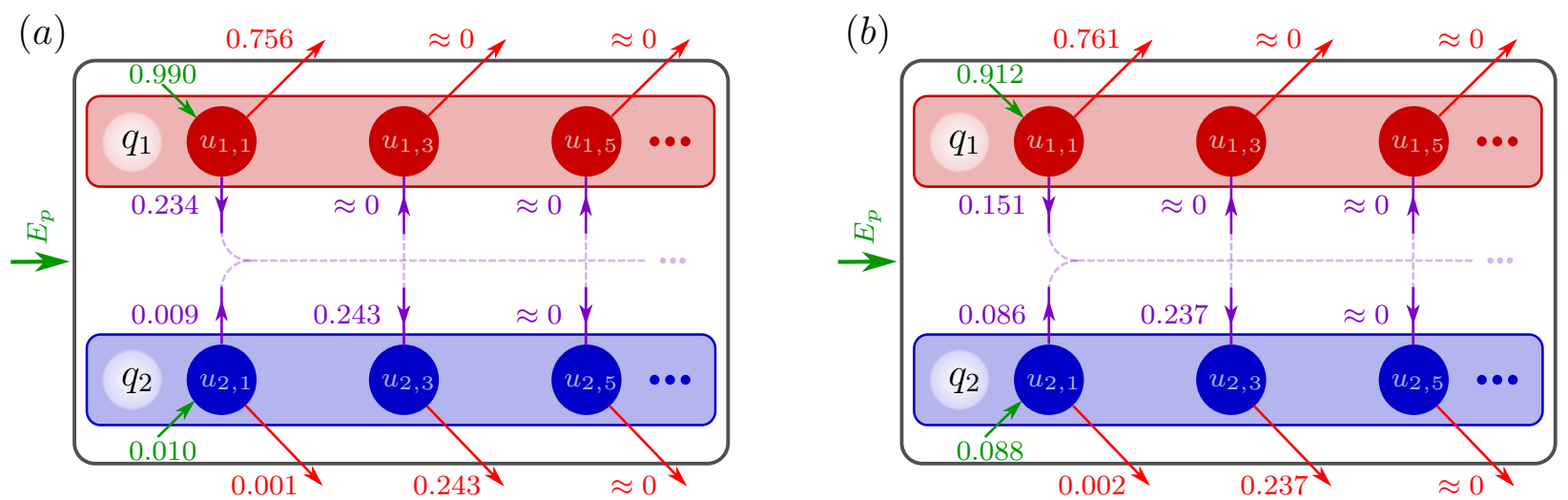

Fig. 4 Energy-transfer balancing between harmonics of the first two modes of the cantilever beam. The thin red, green and purple arrows represent the net periodic energy transfer in and out the harmonics, $E_{D i, j}, E_{P i, j}$ and $E_{N i, j}$ respectively, where the values show the ratio of energy transfer terms over total energy input $E_{p}$. (a) The periodic energy transfer for the forced response, labelled with a cross, on the isolated forced responses in Fig. 3(a) (i.e. the forcing location is at $\left.L_{1}=0.7 L\right)$. (b) The periodic energy transfer for the forced response, labelled with a cross, on the isolated forced responses in Fig. 3(b) (i.e. the forcing location is at $L_{1}=0.2 L$ )

terms, occurs between fundamental components of the two modes, $u_{1,1}$ and $u_{2,3}$, where the transferred energy is lost via damping. Note that the NNM being considered has a $1: 3$ response so $u_{2,3}$ is the fundamental component of the $2^{\text {nd }}$ mode response. As such, ignoring the small contributions from harmonics, the energy balancing, Eq. (11), can be approximately captured by the fundamental components. This also gives rise to the accurate predictions via META (see Fig. 3(a)), where the responses of the fundamental components are used to represent the two modes.

For the case in Fig. 4(b) where the excitation is at $L_{1}=0.2 L$, the major energy input from external forcing is still imparted to $u_{1,1}$, as with the case in Fig. 4(a); however, with an increased amount given to $u_{2,1}$ (about nine percent of the total energy input). As for the internal energy transfer between harmonics, $E_{N i, j}$, besides the fundamental components, a significant involvement of $u_{2,1}$ can also be observed. As such, without considering the energy transfer arising from harmonics $\left(u_{2,1}\right.$ for this case), a significant error in energy balancing analysis via META is shown in Fig. 3(b), which fails to capture the resonances on the isolated forced responses.

By comparing cases in Fig. 3, the limitations of META can be explained by considering the energy transfer between harmonics. It also demonstrates the importance of considering multiple harmonics in energy transfer analysis - the topic of this paper. To establish energy balancing analysis considering multiple harmonics, the mode-level energy balancing, i.e. Eqs. (5) and (11), is extended to the harmonic level.

\subsection{Energy-transfer balancing at the harmonic level}

As with the extension of energy balancing analysis from the system level to the mode level, it can be further extended to the harmonic level, where a similar mechanism holds - for a periodic response, the net energy transfer in and out of any harmonic must be zero, i.e

$E_{D i, j}+E_{P i, j}+E_{N i, j}=0$.

This may be seen as the fundamental level as it is established using the fundamental elements - harmonics, and describes the fundamental mechanism of energy transfer of nonlinear systems during periodic responses.

At the harmonic level, for an NNM, the net energy transfer between harmonics must be zero, i.e. $E_{N i, j}=$ 0 . Therefore, the relationship between NNMs and forced responses can be obtained from Eq. (13), which reads

$E_{P i, j}=-E_{D i, j}$.

This indicates that the harmonic forcing energy gain is balanced by the harmonic damping energy loss - an extension of Eq. (6) to the harmonic level. Using this relationship, the force appropriation technique, has been proposed to extract NNM branches experimentally [23]. In order to satisfy Eq. (14), a quadrature criterion, where the excitation has to compensate for the damping terms, is achieved by applying $90^{\circ}$ phase-lagged forces that contain all harmonic components of all modes in an NNM.

However, in practice, such a perfect forcing set can never be precisely achieved, which causes the response to deviate from the NNM - a non-zero energy transfer to harmonics, i.e. $E_{N i, j} \neq 0$. Such a non-zero $E_{N i, j}$ may couple all harmonics of all modes within a NNM, and 


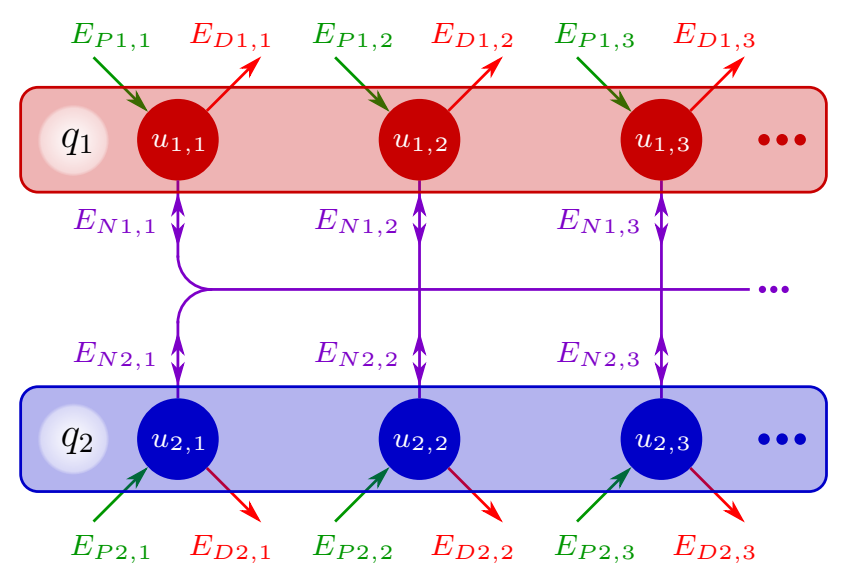

Fig. 5 A schematic of the energy transfer between the harmonics of a two-mode system.

this will be derived analytically in the following discussions. A general energy-transfer schematic for a twomode multi-harmonic system is shown in Fig. 5, which represents an extension of that shown in Fig. 1(b); and is a generalised version of that used for the example we have just discussed, Fig. 4. This illustrates a more complex energy-transfer network among all harmonics at the harmonic level, when compared to taking a higherlevel view at the mode level.

Similar to the mode level, to evaluate the deviation of forced resonances $\left(E_{N i, j} \neq 0\right)$ from NNM solutions $\left(E_{N i, j}=0\right)$, the effect of non-zero $E_{N i, j}$ may be considered. As with the assumption of META, where a modal phase-shift is used to evaluate a non-zero $E_{N i}$, the nonzero $E_{N i, j}$ for the energy balancing at the harmonic level may be evaluated by considering the corresponding harmonic phase-shift. This allows the Harmoniclevel Energy Transfer Analysis (HETA) to be analytically formulated, detailed in the following.

\section{Harmonic-level Energy Transfer Analysis for systems under harmonic forcing}

This section considers the extension of the modelevel energy transfer analysis, proposed in Ref. [12], to consider energy transfer between harmonics. This is achieved by first introducing the assumption that, near resonance, the forced responses may be seen as perturbations from the NNM solutions due to phase-shifts of harmonics. The energy transfer analysis over all harmonics of all modes is then formulated using Eq. (13), which leads to an underdetermined problem, i.e. the unknowns outnumber the equations. To solve this, the force reduction, along with extra phase constraints imposed by the quadrature conditions, are introduced to formulate a determined, or solvable, equation set.
3.1 Harmonic phase-shifts of NNMs under quadrature forcing

To account for multiple harmonics, the displacement of the $i^{\text {th }}$ mode, for an NNM response, is approximated as a sum of a finite number of harmonics, such that

$\bar{q}_{i} \approx \sum_{j \in \mathcal{H}_{i}} \bar{u}_{i, j}=\sum_{j \in \mathcal{H}_{i}} \bar{U}_{i, j} \cos \left(j \omega t+\bar{\phi}_{i, j}\right)$,

where the overbar, $\mathbf{\bullet}$, indicates that this is an NNM response, and where $\bar{u}_{i, j}$ is the $j^{\text {th }}$ harmonic of the $i^{\text {th }}$ modal displacement, $\bar{q}_{i}$; and $\mathcal{H}_{i}$ is the set of harmonics used to approximate $\bar{q}_{i}$. The amplitude and phase of $\bar{u}_{i, j}$ are represented by $\bar{U}_{i, j}$ and $\bar{\phi}_{i, j}$ respectively, and the frequency $\omega$ is defined as $\omega=2 \pi T^{-1}$, where $T$ is the period of the response of the system (i.e. considering all modes).

As with the modal displacements, the external forcing applied to the $i^{\text {th }}$ mode may be separated into harmonic components, written

$p_{i}=\sum_{j \in \mathcal{H}_{i}} p_{i, j}=\sum_{j \in \mathcal{H}_{i}}-P_{i, j} \sin \left(j \omega t+\bar{\phi}_{i, j}\right)$.

Note that this assumes that the harmonics of the excitation force are all in quadrature (i.e. at $\pi / 2$ outof-phase, or $\pi / 2$ phase-lagged) with the corresponding modal harmonic. This also assumes that all harmonics are forced; however, the unforced harmonics may be specified as those where the excitation amplitude, $P_{i, j}$, is zero - this is revisited later in $§ 3.3$.

Following the approach used in [12], as described in $\S 2$, it is assumed that the forced response may be seen as a perturbation from an NNM response with a shift in the phase of the response. Here, however, this phaseshift is applied to all harmonics of the response, rather than just the fundamental component (as considered in [12]). The phase-shift of the $j^{\text {th }}$ harmonic of the $i^{\text {th }}$ mode is written $\hat{\phi}_{i, j}$. Introducing these phase-shifts to Eq. (15), the forced response of the $i^{\text {th }}$ mode is written

$q_{i}=\sum_{j \in \mathcal{H}_{i}} u_{i, j}=\sum_{j \in \mathcal{H}_{i}} U_{i, j} \cos \left(j \omega t+\bar{\phi}_{i, j}+\hat{\phi}_{i, j}\right)$.

Note that, as in [12], it is assumed that all amplitudes in the forced response are equal to those in the NNM $\left(U_{i, j}=\bar{U}_{i, j}\right)$, i.e. the amplitudes are unaffected by the application of forcing and damping.

Based on this phase-shift assumption, we now formulate the energy transfer analysis at the harmonic level via Eq. (13), where the energy-transfer terms, $E_{D i, j}, E_{P i, j}$ and $E_{N i, j}$, may be approximated as linear functions of the excitation amplitude, $P_{i, j}$, and the phase-shifts, $\hat{\phi}_{i, j}$. 
3.2 Problem formulation: energy-transfer balancing at the harmonic level

Equation (13) shows the relationship between the damping, forcing and nonlinear energy-transfer terms for the $j^{\text {th }}$ harmonic of the $i^{\text {th }}$ mode. These terms may be computed respectively using Eq. (12). In addition, in Appendix A, it is shown that these terms may be written as

$$
\begin{aligned}
& E_{D i, j}=E_{D i, j}^{\dagger}, \\
& E_{P i, j} \approx P_{i, j} E_{P i, j}^{\dagger}, \\
& E_{N i, j} \approx \sum_{n=1}^{N} \sum_{k \in \mathcal{H}_{n}} E_{N i, j}^{\dagger(n, k)} \hat{\phi}_{n, k},
\end{aligned}
$$

where the dagger, $\bullet^{\dagger}$, denotes a known term - i.e. a term that may be computed using the NNM response (which is assumed to be known). Whilst the forcing amplitudes, $P_{i, j}$, and phase-shifts, $\hat{\phi}_{n, k}$, are assumed to be unknown. The known terms are found using

$$
\begin{aligned}
E_{D i, j}^{\dagger}= & \pi j^{2} \omega d_{i} U_{i, j}^{2}, \\
E_{P i, j}^{\dagger}= & -\pi j U_{i, j}, \\
E_{N i, j}^{\dagger(n, k)}= & \left\{\begin{array}{c}
\frac{1}{k \omega} \int_{0}^{T} \frac{\partial N_{q i}}{\partial q_{n}} \dot{\bar{u}}_{i, j} \dot{\bar{u}}_{n, k} \mathrm{~d} t \\
\text { when: }\{i, j\} \neq\{n, k\} \\
\frac{1}{j \omega} \int_{0}^{T} \frac{\partial N_{q i}}{\partial q_{i}} \dot{\bar{u}}_{i, j}^{2} \mathrm{~d} t \\
+\frac{1}{j \omega} \int_{0}^{T} N_{q i} \ddot{\bar{u}}_{i, j} \mathrm{~d} t, \\
\text { when: }\{i, j\}=\{n, k\}
\end{array}\right.
\end{aligned}
$$

Equation (18b) shows that the energy transferred to the $j^{\text {th }}$ harmonic of the $i^{\text {th }}$ mode from external forcing is only due to the force directly applied to that harmonic (i.e. it is only a function of $P_{i, j}$ ), of which examples are shown in Fig. 4. The nonlinear counterpart, Eq. (18c), represents the nonlinear energy transfer from all harmonics of all modes in an NNM to the $j^{\text {th }}$ harmonic of the $i^{\text {th }}$ mode; and it reveals that a phase-shift in any harmonic of any mode, i.e. the $k^{\text {th }}$ harmonic of the $n^{\text {th }}$ mode, may lead to an energy transfer to the $j^{\text {th }}$ harmonic of the $i^{\text {th }}$ mode. This demonstrates that any nonlinear energy-transfer term may couple all harmonics of all modes in an NNM, as shown in Fig. 5.

Substituting the expressions for these terms, Eqs. (18), into the energy balancing expression, Eq. (13), gives

$E_{D i, j}^{\dagger}+P_{i, j} E_{P i, j}^{\dagger}+\sum_{n=1}^{N} \sum_{k \in \mathcal{H}_{n}} E_{N i, j}^{\dagger(n, k)} \hat{\phi}_{n, k}=0$.

By collecting the energy-transfer terms due to damping, forcing and nonlinearity, the energy balancing can be formulated from the $j^{\text {th }}$ harmonic, to all harmonics of the $i^{\text {th }}$ mode, and to all modes of the system, summarised in Table 2, where the total number of harmonics used to represents all $N$ modes of the system is given by

$R_{\mathcal{H}}=\sum_{n=1}^{N}\left|\mathcal{H}_{n}\right|$

and where the damping energy vector, $\mathbf{E}_{D}^{\dagger}$, the forcing amplitude vector, $\mathbf{P}$, and the harmonic phase-shifts vector, $\hat{\boldsymbol{\phi}}$, measure $\left\{R_{\mathcal{H}} \times 1\right\}$; the forcing energy coefficient matrix, $\mathbf{E}_{P}^{\dagger}$ is a square, diagonal matrix measuring $\left\{R_{\mathcal{H}} \times R_{\mathcal{H}}\right\}$; and the nonlinear energy-transfer coefficient matrix, $\mathbf{E}_{N}^{\dagger}$, is a $\left\{R_{\mathcal{H}} \times R_{\mathcal{H}}\right\}$ matrix, populated with $E_{N i, j}^{\dagger(n, k)}$.

Up to this point, the energy transfer analysis is formulated as a set of $R_{\mathcal{H}}$ equations, given by Eq. (26), with $2 R_{\mathcal{H}}$ unkonwns, consisting of $R_{\mathcal{H}}$ phase-shifts, $\hat{\phi}_{n, k}$, and $R_{\mathcal{H}}$ forcing amplitudes, $P_{i, j}$. To solve this underdetermined equation set, where the unknowns outnumber the equations, either reducing the number of unknowns, introducing extra constraints, or both, are needed.

\subsection{Problem solving: quadrature harmonic forcing}

As previously discussed, the current formulation allows all harmonics of all modes to be forced. However, in practical applications, the forcing can only be applied to a limited number of harmonics. Here, a special case is considered where a limited number of quadrature harmonic forces ${ }^{2}$ are applied; whilst the more complex case, where quadrature physical forces are applied, will be discussed later in $\S 4$. To specify this here the harmonics of the non-zero forcing (applied to the $i^{\text {th }}$ mode) are defined as belonging to the set $\tilde{\mathbf{P}}_{i}$, i.e. the non-zero subset of $\mathbf{P}_{i}$. As such, the total number of non-zero forcing applied to the system is given by

$R_{\mathcal{F}}=\sum_{i=1}^{N}\left|\tilde{\mathbf{P}}_{i}\right|$.

With this, $\mathbf{P}$ may be simplified by discarding the zerovalued elements to reduce the number of unknowns that need to be estimated. This reduction is achieved by relating the vector of non-zero forcing amplitudes $\tilde{\mathbf{P}}$ (measuring $\left\{R_{\mathcal{F}} \times 1\right\}$ ) to $\mathbf{P}$ via the force reduction $m a$ trix, $\mathbf{C}_{P}$, where

$\mathbf{P}=\mathbf{C}_{P} \tilde{\mathbf{P}}$.

2 Here the term quadrature harmonic forcing indicates that the applied harmonic forcing is in quadrature with the corresponding harmonic displacement, as defined in Eq. (16). 
Table 2 Harmonic-level energy balancing.

\begin{tabular}{|c|c|c|}
\hline Energy balancing for the $j^{\text {th }}$ harmonic of the $i^{\text {th }}$ mode & Expressions for coefficients & \\
\hline$E_{D i, j}^{\dagger}+E_{P i, j}^{\dagger} P_{i, j}+\mathbf{E}_{N i, j}^{\dagger} \hat{\boldsymbol{\phi}}=0$ & $\begin{aligned} \mathbf{E}_{N i, j}^{\dagger(n)} & =\left[\begin{array}{llllll}E_{N i, j}^{\dagger(n, 1)} & E_{N i, j}^{\dagger(n, 2)} & \ldots & E_{N i, j}^{\dagger(n, k)} & \ldots & E_{N i, j}^{\dagger(n, K)}\end{array}\right] \\
\hat{\phi}_{n} & =\left(\begin{array}{llllll}\hat{\phi}_{n, 1} & \hat{\phi}_{n, 2} & \ldots & \hat{\phi}_{n, k} & \ldots & \hat{\phi}_{n, K}\end{array}\right)^{\top} \\
\mathbf{E}_{N i, j}^{\dagger} & =\left[\begin{array}{llllll}\mathbf{E}_{N i, j}^{\dagger(1)} & \mathbf{E}_{N i, j}^{\dagger(2)} & \ldots & \mathbf{E}_{N i, j}^{\dagger(n)} & \ldots & \mathbf{E}_{N i, j}^{\dagger(N)}\end{array}\right] \\
\hat{\phi} & =\left(\begin{array}{llllll}\hat{\phi}_{1}^{\top} & \hat{\phi}_{2}^{\top} & \ldots & \hat{\phi}_{n}^{\top} & \ldots & \hat{\phi}_{N}^{\top}\end{array}\right)^{\top}\end{aligned}$ & $\begin{array}{l}(23 \mathrm{a}) \\
(23 \mathrm{~b}) \\
(23 \mathrm{c}) \\
(23 \mathrm{~d})\end{array}$ \\
\hline Energy balancing for all harmonics of the $i^{\text {th }}$ mode & Expressions for coefficients & \\
\hline $\mathbf{E}_{D i}^{\dagger}+\mathbf{E}_{P i}^{\dagger} \mathbf{P}_{i}+\mathbf{E}_{N i}^{\dagger} \hat{\boldsymbol{\phi}}=\mathbf{0}$ & 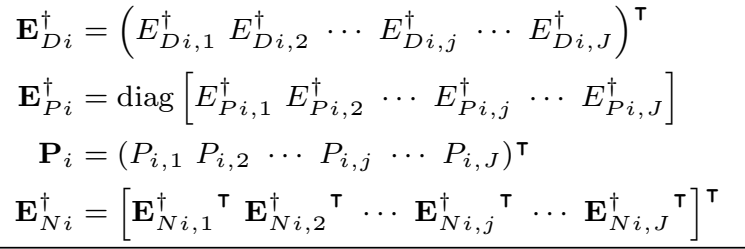 & $\begin{array}{l}(25 \mathrm{a}) \\
(25 \mathrm{~b}) \\
(25 \mathrm{c}) \\
(25 \mathrm{~d})\end{array}$ \\
\hline Energy balancing for all modes of the system & Expressions for coefficients & \\
\hline $\mathbf{E}_{D}^{\dagger}+\mathbf{E}_{P}^{\dagger} \mathbf{P}+\mathbf{E}_{N}^{\dagger} \hat{\boldsymbol{\phi}}=\mathbf{0}$ & 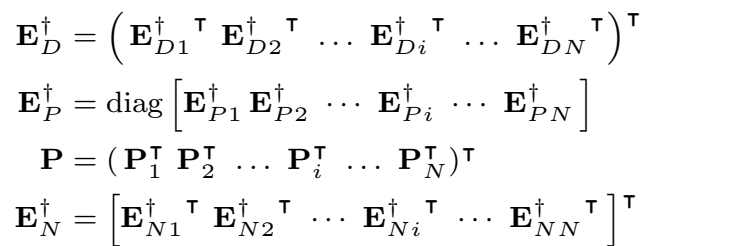 & $\begin{array}{l}(27 a) \\
(27 b) \\
(27 c) \\
(27 d)\end{array}$ \\
\hline
\end{tabular}

The force reduction matrix may be constructed using a $\left\{R_{\mathcal{H}} \times R_{\mathcal{H}}\right\}$ identity matrix and removing the columns associated with unforced harmonics. Hence, $\mathbf{C}_{P}$ is of size $\left\{R_{\mathcal{H}} \times R_{\mathcal{F}}\right\}$. Substituting Eq. (29) into Eq. (26) leads to

$\mathbf{E}_{D}^{\dagger}+\tilde{\mathbf{E}}_{P}^{\dagger} \tilde{\mathbf{P}}+\mathbf{E}_{N}^{\dagger} \hat{\boldsymbol{\phi}}=\mathbf{0}$

where $\tilde{\mathbf{E}}_{P}^{\dagger}=\mathbf{E}_{P}^{\dagger} \mathbf{C}_{P}$ is also used, and hence $\tilde{\mathbf{E}}_{P}^{\dagger}$ measures $\left\{R_{\mathcal{H}} \times R_{\mathcal{F}}\right\}$. Through force reduction, the total number of unknowns is now reduced to $\left(R_{\mathcal{H}}+R_{\mathcal{F}}\right)$, i.e. $R_{\mathcal{H}}$ phase-shifts and $R_{\mathcal{F}}$ forcing amplitudes. However, it is still underdetermined and additional $R_{\mathcal{F}}$ constraints are required to solve the equations.

As it is assumed that all forces are in quadrature with the harmonic they are forcing, it may also be assumed that the forced harmonics do not exhibit a phase-shift ${ }^{3}$. This condition may be enforced using a phase constraint matrix, $\mathbf{C}_{\phi}$, where

$\mathbf{C}_{\phi} \hat{\boldsymbol{\phi}}=\mathbf{0}$.

Here, $\mathbf{C}_{\phi}$ is a $\left\{R_{\mathcal{F}} \times R_{\mathcal{H}}\right\}$ matrix that constrains the phase-shifts of all forced harmonics to be zero. In the case where all harmonic forces are independent, the phase constraint matrix is the transpose of the force

3 If the forced harmonics did exhibit a phase-shift, they would no longer be in quadrature. reduction matrix, i.e. $\mathbf{C}_{\phi}=\mathbf{C}_{P}^{\top}$; hence $\mathbf{C}_{\phi}$ may be constructed by removing the rows associated with unforced harmonic of a $\left\{R_{\mathcal{H}} \times R_{\mathcal{H}}\right\}$ identity matrix. The phase constraints introduce $R_{\mathcal{F}}$ equations, and hence combining Eq. (31) with the energy balancing expressions, defined by Eq. (30), leads the harmonic-level energy transfer analysis to a determined, or solvable, equation set.

Equations (30) and (31) are now combined and written into matrix form as

$\mathbf{A} \mathbf{v}=\mathbf{B}$

where

$\mathbf{v}=\left(\begin{array}{c}\hat{\boldsymbol{\phi}} \\ \tilde{\mathbf{P}}\end{array}\right), \quad \mathbf{A}=\left[\begin{array}{cc}\mathbf{E}_{N}^{\dagger} & \tilde{\mathbf{E}}_{P}^{\dagger} \\ \mathbf{C}_{\phi} & \mathbf{0}\end{array}\right], \quad \mathbf{B}=\left(\begin{array}{c}-\mathbf{E}_{D}^{\dagger} \\ \mathbf{0}\end{array}\right)$

and where the unknowns, i.e. $\hat{\boldsymbol{\phi}}$ and $\tilde{\mathbf{P}}$, are collected in the vector $\mathbf{v}$, and the known coefficients are collected in matrices $\mathbf{A}$ and $\mathbf{B}$. As the number of constraints now matches the number of unknowns, the unknown terms are found using

$\mathbf{v}=\mathbf{A}^{-1} \mathbf{B}$.

This allows the phase-shifts of any unforced harmonics and the amplitudes of any forces, which are represented in $\mathbf{v}$, to be computed. 
For the case where the forces applied to the harmonics are independent, the use of the forcing reduction matrix, $\mathbf{C}_{P}$, and the phase constraint matrix, $\mathbf{C}_{\phi}$, could be viewed as unnecessary: $\tilde{\mathbf{E}}_{P}^{\dagger}$ may be constructed directly by removing the columns of $\mathbf{E}_{P}^{\dagger}$ that correspond to the unforced harmonics and a lower-dimension problem could be formulated by simply removing the forced phase-shifts and their corresponding columns in $\mathbf{E}_{N}^{\dagger}$. However, this approach allows additional constraints to be introduced, as shown later in $\S 4$. The implementation of this method is now demonstrated using a simple example system.

\subsection{Example 1: a quadrature harmonic forcing case}

To demonstrate the HETA, the cantilever beam, schematically shown in Fig. 2, is again considered. To simplify this case, a single-harmonic force is applied to each mode independently, such that the equations of motion are written

$\ddot{q}_{1}+d_{1} \dot{q}_{1}+\omega_{n 1}^{2} q_{1}+N_{q 1}\left(q_{1}, q_{2}\right)=F_{1} \cos (\Omega t)$,

$\ddot{q}_{2}+d_{2} \dot{q}_{2}+\omega_{n 2}^{2} q_{2}+N_{q 2}\left(q_{1}, q_{2}\right)=F_{2} \cos (3 \Omega t)$,

where the nonlinear forces are given by Eq. (9). Note that the case where forcing is applied to the part-span will be revisited later in $\S 4$.

The parameters of the system are the same as considered in $\S 2.3$, given in Table 1 . It should be noted that the forcing amplitudes, $F_{1}$ and $F_{2}$, are assumed to be unknown (and to be found using HETA). Also note that the linear natural frequencies have a ratio of approximately $1: 3$, i.e. $\omega_{n 2} / \omega_{n 1} \approx 3$. This leads to a $1: 3$ modal interaction, as discussed in detail in [32].

Before applying HETA, the NNM branches of the system need to be obtained to provide known parameters to compute the coefficients in Eq. (34). Regarding the definition of an NNM, i.e. a periodic response of a conservative system [18], the NNM branches can be computed by removing the external forcing and damping terms in Eq. (35). For comparison, the forced responses, obtained via quadrature forcing, or force appropriation technique [23], are also presented. The first NNM branch is presented in Fig. 6 by a solid-blue line. The top and bottom panels of Fig. 6 show the maximum displacement amplitudes of the first and second modes respectively. In both of these panels, a distinctive loop region is seen, indicating a strong internal resonance between the two linear modes. Further discussion of this NNM branch can be found in [32]. The thin red lines, with dots, in Fig. 6 show the responses of the system when forcing is in quadrature. For the system described by Eq. (35), quadrature is achieved when the
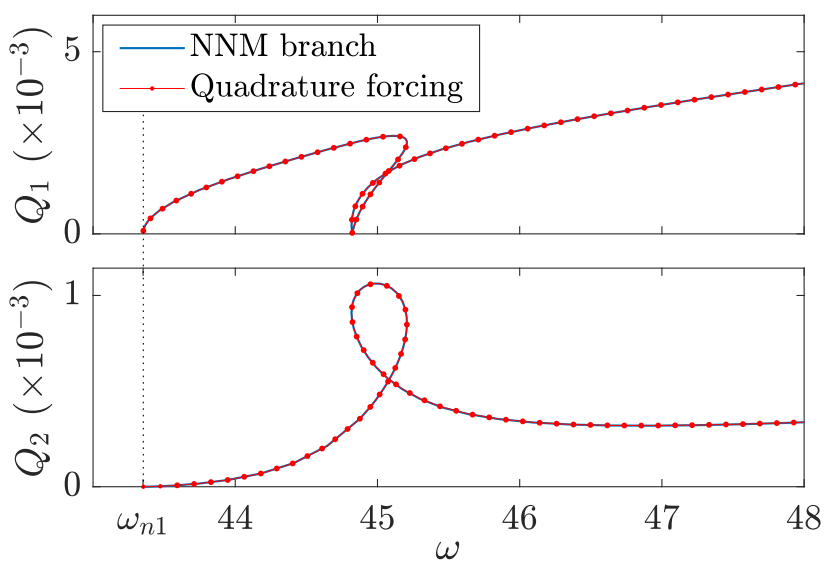

Fig. 6 The first NNM branch (blue line) and quadrature branch (red line with dots) for the two-mode beam. The top panel is in the projection of the response frequency, $\omega$, against the maximum displacement amplitude of the first linear mode, $Q_{1}$. The bottom panel is in the projection of $\omega$ against the maximum displacement amplitude of the second mode, $Q_{2}$. The dotted-black line denotes the first linear natural frequency.

force applied to the first mode is at $90^{\circ}$ to the displacement of the first harmonic, $u_{1,1}$, and when the second modal forcing is at $90^{\circ}$ to the displacement of the third harmonic, $u_{2,3}-$ see $[23,28]$ for further details. As the NNMs and the forced responses appear to be close, it is expected that the phase-shifts will be small.

To apply HETA, a finite number of harmonics must be used to approximate the modal displacements, as previously discussed in Eq. (15). For this example, it is assumed that the first and third harmonics of each mode are sufficient ${ }^{4}$, i.e.

$\mathcal{H}_{1}=\{1,3\}, \quad \mathcal{H}_{2}=\{1,3\}$,

such that

$q_{1} \approx u_{1,1}+u_{1,3}, \quad q_{2} \approx u_{2,1}+u_{2,3}$.

Whilst additional harmonics would provide greater accuracy, just two harmonics are considered here for simplicity.

To construct the energy balancing equations using Eq. (30), the coefficients and unknowns are first assembled into vector/matrix form. The damping energy vector is defined, using Eqs. (19a), (25a) and (27a), as

$\mathbf{E}_{D}^{\dagger}=\left(\begin{array}{l}E_{D 1,1}^{\dagger} \\ E_{D 1,3}^{\dagger} \\ E_{D 2,1}^{\dagger} \\ E_{D 2,3}^{\dagger}\end{array}\right)=\left(\begin{array}{c}\pi \omega d_{1} U_{1,1}^{2} \\ 9 \pi \omega d_{1} U_{1,3}^{2} \\ \pi \omega d_{2} U_{2,1}^{2} \\ 9 \pi \omega d_{2} U_{2,3}^{2}\end{array}\right)$.

\footnotetext{
4 Note that this system contains only cubic nonlinearities see Eqs. (9). As such, only odd-numbered harmonics will be present in the resonant response.
} 
Next, using Eqs. (25b), (25c), (27b) and (27c), the matrix of forcing energy coefficients, and vector of unknown harmonic forcing amplitudes, are written as

$\mathbf{E}_{P}^{\dagger}=\left[\begin{array}{cccc}E_{P 1,1}^{\dagger} & 0 & 0 & 0 \\ 0 & E_{P 1,3}^{\dagger} & 0 & 0 \\ 0 & 0 & E_{P 2,1}^{\dagger} & 0 \\ 0 & 0 & 0 & E_{P 2,3}^{\dagger}\end{array}\right], \quad \mathbf{P}=\left(\begin{array}{c}P_{1,1} \\ P_{1,3} \\ P_{2,1} \\ P_{2,3}\end{array}\right)$.

As the third harmonic of the first mode and the first harmonic of the second mode are unforced, the force reduction matrix and reduced vector of forcing amplitudes may be written

$\mathbf{C}_{P}=\left[\begin{array}{ll}1 & 0 \\ 0 & 0 \\ 0 & 0 \\ 0 & 1\end{array}\right], \quad \tilde{\mathbf{P}}=\left(\begin{array}{l}P_{1,1} \\ P_{2,3}\end{array}\right)=\left(\begin{array}{c}F_{1} \\ F_{2}\end{array}\right)$,

where the forcing amplitudes $P_{1,1}=F_{1}$ and $P_{2,3}=F_{2}$, from the equations of motion, i.e. Eq. (35), have been substituted. Using the force reduction matrix, the reduced forcing energy coefficient matrix is given by

$\tilde{\mathbf{E}}_{P}^{\dagger}=\mathbf{E}_{P}^{\dagger} \mathbf{C}_{P}=\left[\begin{array}{cc}E_{P 1,1}^{\dagger} & 0 \\ 0 & 0 \\ 0 & 0 \\ 0 & E_{P 2,3}^{\dagger}\end{array}\right]=\left[\begin{array}{cc}-\pi U_{1,1} & 0 \\ 0 & 0 \\ 0 & 0 \\ 0 & -3 \pi U_{1,3}\end{array}\right]$,

where Eq. (19b) has been used to define the forcing energy coefficients.

Next, as used in Eqs. (23a), (23c), (25d) and (27d), the matrix of nonlinear coefficients is written

$\mathbf{E}_{N}^{\dagger}=\left[\begin{array}{llll}E_{N 1,1}^{\dagger(1,1)} & E_{N 1,1}^{\dagger(1,3)} & E_{N 1,1}^{\dagger(2,1)} & E_{N 1,1}^{\dagger(2,3)} \\ E_{N 1,1}^{\dagger(1,1)} & E_{N 1,3}^{\dagger(1,3} & E_{N 1,3}^{\dagger(2,1)} & E_{N 1,3}^{\dagger(2,3)} \\ E_{N 2,1}^{\dagger(1,1)} & E_{N 2,3}^{\dagger(1,3} & E_{N 2,1}^{\dagger(2,1)} & E_{N 2,1}^{\dagger(2,3)} \\ E_{N 2,3}^{\dagger(1,1)} & E_{N 2,3}^{\dagger(1,3)} & E_{N 2,3}^{\dagger(2,1)} & E_{N 2,3}^{\dagger(2,3)}\end{array}\right]$,

where the elements of this matrix are computed using Eq. (19c).

Finally, the phase constraint matrix and vector of phase-shifts are written

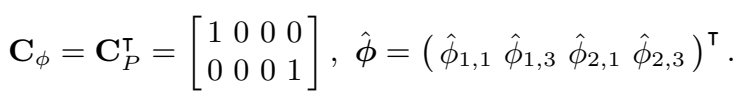

Using the expressions from (38) to (43), the components to apply harmonic-level energy transfer analysis, i.e. Eq. (34), may be collected as

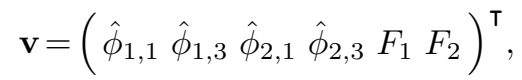

$$
\begin{aligned}
& \mathbf{B}=\left(-E_{D 1,1}^{\dagger}-E_{D 1,3}^{\dagger}-E_{D 2,1}^{\dagger}-E_{D 2,3}^{\dagger} 00\right)^{\top}, \\
& \mathbf{A}=\left[\begin{array}{cccccc}
E_{N 1,1}^{\dagger(1,1)} & E_{N 1,1}^{\dagger(1,3)} & E_{N 1,1}^{\dagger(2,1)} & E_{N 1,1}^{\dagger(2,3)} & E_{P 1,1}^{\dagger} & 0 \\
E_{N 1,3}^{\dagger(1,1)} & E_{N 1,3}^{\dagger(1,3)} & E_{N 1,3}^{\dagger(2,1)} & E_{N 1,3}^{\dagger(2,3)} & 0 & 0 \\
E_{N 2,1}^{\dagger(1,1)} & E_{N 2,1}^{\dagger(1,3)} & E_{N 2,1}^{\dagger(2,1)} & E_{N 2,1}^{\dagger(2,3)} & 0 & 0 \\
E_{N 2,3}^{\dagger(1,1)} & E_{N 2,3}^{\dagger(1,3)} & E_{N 2,3}^{\dagger(2,1)} & E_{N 2,3}^{\dagger(2,3)} & 0 & E_{P 2,3}^{\dagger} \\
1 & 0 & 0 & 0 & 0 & 0 \\
0 & 0 & 0 & 1 & 0 & 0
\end{array}\right] .
\end{aligned}
$$

Noting that all components in $\mathbf{A}$ and $\mathbf{B}$ may be computed using an NNM solution. The vector of unknown phase-shifts and forcing amplitudes may now be computed using Eq. (34), i.e. $\mathbf{v}=\mathbf{A}^{-1} \mathbf{B}$.

Figure 7 shows the forcing amplitudes, $\left|F_{1}\right|$ and $\left|F_{2}\right|$, and the phase-shifts, $\hat{\phi}_{1,3}$ and $\hat{\phi}_{2,1}$. In all panels of Fig. 7, the computationally-cheap analyticallypredicted values, obtained via HETA, are represented by blue lines, and the computationally-expensive numerically-simulated values, obtained via the force appropriation technique, are shown by red lines with dots. The difference between the analytically-predicted and numerically-simulated forcing amplitudes, $\left|F_{1}\right|$ and $\left|F_{2}\right|$, is indistinguishable, showing that these have been predicted with a very high level of accuracy. Although there is some discrepancy, the phase-shift values, $\hat{\phi}_{1,3}$ and $\hat{\phi}_{2,1}$, also show a very good agreement, despite the low number of harmonics used to approximate these responses. The greatest inaccuracy can be seen near the linear natural frequency, $\omega_{n 1}$, (where NNMs are close to the linear response) in the $\hat{\phi}_{1,3}$ and $\hat{\phi}_{2,1}$ phase-shifts. This is likely due to numerical error in both the simulated and estimated values, as the harmonics are very small for low-amplitude NNMs. Additionally, as NNMs approach the linear case, the mixed-mode NNM response approaches a linear single-mode response whose phase is no longer defined.

To summarise, the results of HETA, shown in blue in Fig. 7 , have been estimated via the procedure outlined in this section using only the NNM responses. For the sake of simplicity, this approach has assumed that the response consists of just two harmonics, which limits the accuracy of the predictions. However, owing to the semi-analytical framework, it is convenient to increase the number of harmonics without adding much computational effort. As such, this provides a more efficient technique, whilst preserving the necessary high resolution, for resonance predictions when compared with computationally expensive numerical analysis. The following section will consider additional, more complex, examples and demonstrate the application of this approach to cases with a greater number of parameters.

\section{Accounting for quadrature physical forcing}

The formulation of HETA presented in $\S 3$ is restricted to cases where independent harmonic forces are applied to each mode of the system; however, in many practical applications, a force may be applied to multiple modes simultaneously. For example, a force applied to the midspan of a beam is able to excite all modes of the system simultaneously (aside from those that have a node at the forcing location). If one excitation force is applied 
$(a)$

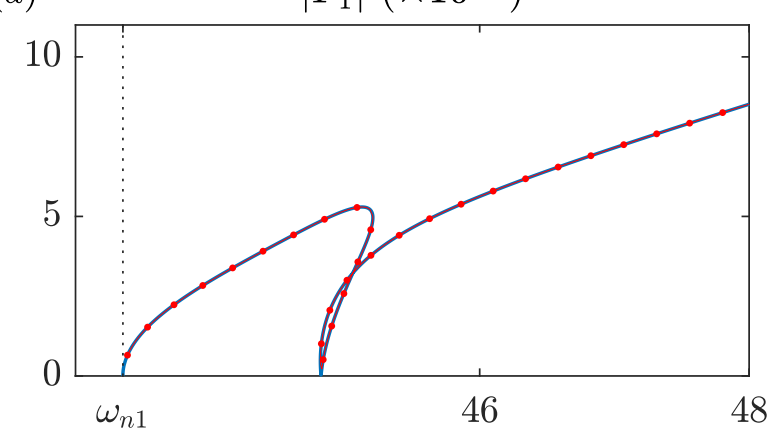

$(c)$

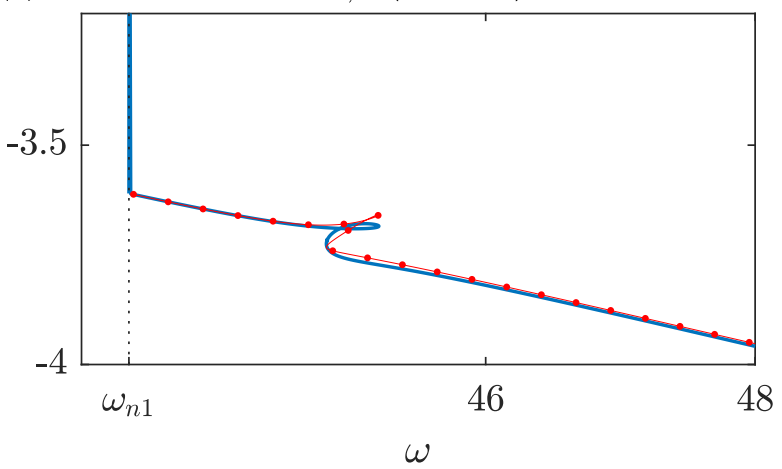

(b)

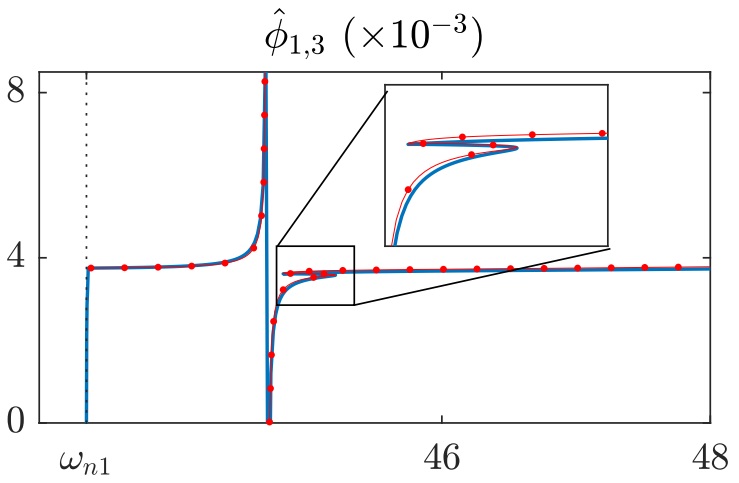

$(d)$

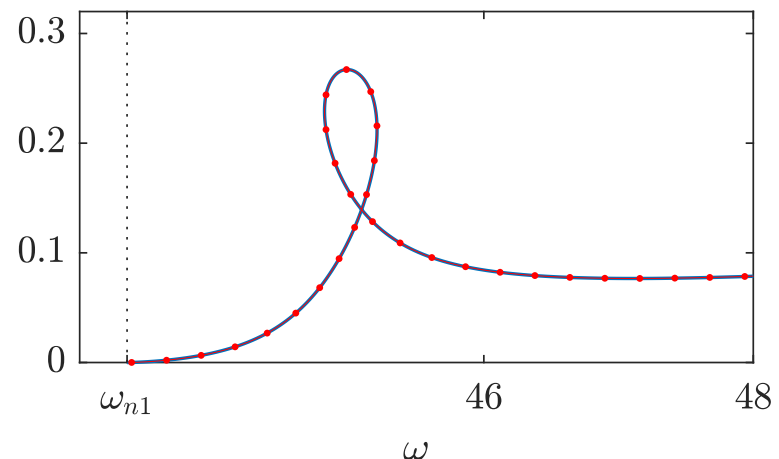

Fig. 7 A comparison between the predictions of HETA (using a two-harmonic approximation for each mode) and the numerically-simulated forced responses. Panels $(a)$ and $(d)$ show the frequency, $\omega$, against the forcing amplitudes $\left|F_{1}\right|$ and $\left|F_{2}\right|$ respectively. Panels $(b)$ and $(c)$ show the frequency, $\omega$, against the phase-shift parameters, $\hat{\phi}_{1,3}$ and $\hat{\phi}_{2,1}$ respectively. In all panels, the blue lines represent analytically-predicted values (using the NNM data), the red lines (with dots) represent the numerical results and the dotted-black line denotes the first linear natural frequency.

to multiple modes, the forcing cannot be in quadrature with all forced modal responses, and hence the phase constraints used in $\S 3$ (i.e. the forced harmonics exhibit zero phase-shifts) become invalid; instead, the modes must be free to exhibit different phase-shifts. In this section, it is shown that HETA may be extended to account for additional forcing conditions - namely where a force is in quadrature with a displacement at a physical location, rather than a harmonic of a mode. This is reflective of most practical scenarios where a force is tuned to reach quadrature with the excitation point. Here, such a forcing is termed a quadrature physical forcing, in comparison to the quadrature harmonic forcing considered in $§ 3$.

\subsection{Formulation of HETA considering quadrature} physical forcing

To formulate HETA with quadrature physical forces, a multi-harmonic force, $\tilde{f}_{\ell}$, applied to the system at location $\ell$, is considered firstly; whilst cases with multiple excitation points will be discussed in the following. Here, the harmonic components of the forcing are defined as belonging to the set $\mathcal{F}_{\ell}$, whose number of elements is $R_{\mathcal{F}_{\ell}}$. Note that it is assumed that the forced harmonics are included in all modes, i.e.

$\mathcal{F}_{\ell} \subseteq \bigcap_{n=1}^{N} \mathcal{H}_{n}$

The $i^{\text {th }}$ modal forcing, $\tilde{p}_{\ell, i}$, can be obtained by introducing the linear modal transform to the physical forcing, and is given by

$\tilde{p}_{\ell, i}=\theta_{\ell, i} \tilde{f}_{\ell}(t)$,

where $\theta_{\ell, i}$ denotes the modeshape of the $i^{\text {th }}$ mode at the excitation point, $\ell$. Separating into harmonic components, $\tilde{f}_{\ell}$ can be expressed as

$\tilde{f}_{\ell}=\sum_{j \in \mathcal{F}_{\ell}} \tilde{f}_{\ell, j}=\sum_{j \in \mathcal{F}_{\ell}}-\tilde{F}_{\ell, j} \sin \left(j \omega t+\tilde{\psi}_{\ell, j}\right)$,

where $\tilde{F}_{\ell, j}$ and $\tilde{\psi}_{\ell, j}$ are the amplitude and phase of the harmonic forcing, $\tilde{f}_{\ell, j}$. As such, the modal forcing, $\tilde{p}_{\ell, i}$, may be approximated as a sum of harmonics using Eqs. (46) and (47), i.e.

$\tilde{p}_{\ell, i}=\sum_{j \in \mathcal{F}_{\ell}}-\theta_{\ell, i} \tilde{F}_{\ell, j} \sin \left(j \omega t+\tilde{\psi}_{\ell, j}\right)$, 
of which the $j^{\text {th }}$ harmonic component is given by

$\tilde{p}_{\ell, i, j}=-\theta_{\ell, i} \tilde{F}_{\ell, j} \sin \left(j \omega t+\tilde{\psi}_{\ell, j}\right)$.

The energy transferred via the modal forcing, $\tilde{p}_{\ell, i}$, to the $j^{\text {th }}$ harmonic of the $i^{\text {th }}$ mode, $u_{i, j}$, can be computed via Eq. (12b), given by

$\tilde{E}_{P \ell, i, j}=-\int_{0}^{T} \tilde{p}_{\ell, i} \dot{u}_{i, j} \mathrm{~d} t \approx-\pi j \phi_{d i, j} U_{i, j} \theta_{\ell, i} \tilde{F}_{\ell, j}$,

where

$\phi_{d i, j}=\cos \left(\tilde{\psi}_{\ell, j}-\bar{\phi}_{i, j}\right)$.

The details of this derivation are given in Appendix A. The phase relationships between the external forcing and NNMs is captured by $\phi_{d i, j}$. In addition, the sign of $\phi_{d i, j}$ accounts for the effect of external forcing on energy transfer - either an energy gain or an energy loss, reflective of the fact that one force may affect modes differently. In contrast, for the case where a harmonic forcing in quadrature is applied, as discussed in $\S 3$, the applied force must lead to a forcing energy-gain term for the enforced harmonic - see Eqs. (18b) and (19b). As shown in Appendix A, $\phi_{d i, j}$ may be related to NNM solutions via quadrature conditions; and hence it is seen as a known parameter.

As discussed in $§ 3.2$, the forcing energy-transfer terms can be separated into forcing energy coefficients, $\tilde{E}_{P \ell, i, j}^{\dagger}$, and harmonic forcing amplitudes, $\tilde{P}_{\ell, i, j}$, i.e.

$\tilde{E}_{P \ell, i, j} \approx \tilde{E}_{P \ell, i, j}^{\dagger} \tilde{P}_{\ell, i, j}$,

where

$\tilde{E}_{P \ell, i, j}^{\dagger}=-\pi j \phi_{d i, j} U_{i, j} \quad$ and $\quad \tilde{P}_{\ell, i, j}=\theta_{\ell, i} \tilde{F}_{\ell, j}$.

Combining the forcing amplitudes for all harmonics of the $i^{\text {th }}$ mode gives

$\tilde{\mathbf{P}}_{\ell, i}=\boldsymbol{\theta}_{\ell, i} \tilde{\mathbf{F}}_{\ell}$,

where

$$
\begin{aligned}
\tilde{\mathbf{P}}_{\ell, i} & =\left(\begin{array}{llllll}
\tilde{P}_{\ell, i, 1} & \tilde{P}_{\ell, i, 2} & \ldots & \tilde{P}_{\ell, i, j} & \ldots & \tilde{P}_{\ell, i, J}
\end{array}\right)^{\top}, \\
\boldsymbol{\theta}_{\ell, i} & =\operatorname{diag}\left[\begin{array}{llllll}
\theta_{\ell, i} & \theta_{\ell, i} & \ldots & \theta_{\ell, i} & \ldots & \theta_{\ell, i}
\end{array}\right], \\
\tilde{\mathbf{F}}_{\ell} & =\left(\begin{array}{llllll}
F_{\ell, 1} & F_{\ell, 2} & \ldots & F_{\ell, j} & \ldots & F_{\ell, J}
\end{array}\right)^{\top} .
\end{aligned}
$$

Next, collecting modal forcing amplitudes for all enforced modes of the system, gives

$\tilde{\mathbf{P}}_{\ell}=\boldsymbol{\theta}_{\ell} \tilde{\mathbf{F}}_{\ell}$,

where

$\tilde{\mathbf{P}}_{\ell}=\left(\begin{array}{llllll}\tilde{\mathbf{P}}_{\ell, 1}^{\top} & \tilde{\mathbf{P}}_{\ell, 2}^{\top} & \ldots & \tilde{\mathbf{P}}_{\ell, i}^{\top} \ldots & \tilde{\mathbf{P}}_{\ell, N}^{\top}\end{array}\right)^{\top}$,

$\boldsymbol{\theta}_{\ell}=\left[\begin{array}{llllll}\boldsymbol{\theta}_{\ell, 1} & \boldsymbol{\theta}_{\ell, 2} & \ldots & \boldsymbol{\theta}_{\ell, i} & \ldots & \boldsymbol{\theta}_{\ell, N}\end{array}\right]^{\top}$,

As discussed in $\S 3$, the reduced forcing energy coefficient matrix, $\tilde{\mathbf{E}}_{P, \ell}^{\dagger}$, can be formulated via

$\tilde{\mathbf{E}}_{P, \ell}^{\dagger}=\mathbf{E}_{P, \ell}^{\dagger} \mathbf{C}_{P \ell}$, where $\mathbf{E}_{P, \ell}^{\dagger}$ is a $\left\{R_{\mathcal{H}} \times R_{\mathcal{H}}\right\}$ diagonal matrix with leading diagonal elements, $E_{P \ell, i, j}^{\dagger}$, defined in Eq. (53); and the force reduction matrix, $\mathbf{C}_{P \ell}$, may be constructed using a $\left\{R_{\mathcal{H}} \times R_{\mathcal{H}}\right\}$ identity matrix and removing columns associated with unforced harmonics.

The forcing energy-transfer terms are then collected with respect to known and unknown terms, i.e.

$\tilde{\mathbf{E}}_{F \ell}^{\dagger} \tilde{\mathbf{F}}_{\ell}=\mathbf{E}_{P \ell}^{\dagger} \mathbf{C}_{P \ell} \boldsymbol{\theta}_{\ell} \tilde{\mathbf{F}}_{\ell}$

where $\tilde{\mathbf{E}}_{F \ell}^{\dagger}=\mathbf{E}_{P \ell}^{\dagger} \mathbf{C}_{P \ell} \boldsymbol{\theta}_{\ell}$, measuring $\left\{R_{\mathcal{H}} \times R_{\mathcal{F}_{\ell}}\right\}$, and $\tilde{\mathbf{F}}_{\ell}$ is a vector, measuring $\left\{R_{\mathcal{F}_{\ell}} \times 1\right\}$.

Up to this point, $\tilde{\mathbf{E}}_{F \ell}^{\dagger}$ and $\tilde{\mathbf{F}}_{\ell}$ for a single excitation at location $\ell$ are constructed. To account for cases with multiple excitations, the forcing locations are defined as in the set, $\mathcal{L}$, and hence the total number of forcing harmonics is given by

$R_{\mathcal{F}}=\sum_{\ell \in \mathcal{L}} R_{\mathcal{F}_{\ell}}$.

For each forcing location, $\tilde{\mathbf{E}}_{F \ell}^{\dagger}$ and $\tilde{\mathbf{F}}_{\ell}$ may be constructed using the procedure outlined above. Then by collecting all matrices and vectors according to excitation points, one has

$$
\begin{aligned}
\tilde{\mathbf{E}}_{F}^{\dagger} & =\left(\begin{array}{llllll}
\tilde{\mathbf{E}}_{F 1}^{\dagger} & \tilde{\mathbf{E}}_{F 2}^{\dagger} & \ldots & \tilde{\mathbf{E}}_{F \ell}^{\dagger} & \ldots & \tilde{\mathbf{E}}_{F|\mathcal{L}|}^{\dagger}
\end{array}\right), \\
\tilde{\mathbf{F}} & =\left(\begin{array}{lllllll}
\tilde{\mathbf{F}}_{1}^{\top} & \tilde{\mathbf{F}}_{2}^{\top} & \ldots & \tilde{\mathbf{F}}_{\ell}^{\top} & \ldots & \tilde{\mathbf{F}}_{|\mathcal{L}|}^{\top}
\end{array}\right)^{\top},
\end{aligned}
$$

where $|\mathcal{L}|$ denotes the number of forcing locations, $\tilde{\mathbf{E}}_{F}^{\dagger}$ is a $\left\{R_{\mathcal{H}} \times R_{\mathcal{F}}\right\}$ matrix, and $\tilde{\mathbf{F}}$ is a $\left\{R_{\mathcal{F}} \times 1\right\}$ vector.

Other energy-transfer terms, i.e. damping and nonlinear energy-transfer terms, $E_{D i, j}$ and $E_{N i, j}$, are the same as those defined in $\S 3$, and they can be constructed in the same form as $\mathbf{E}_{D}^{\dagger}$ and $\mathbf{E}_{N}^{\dagger}$ respectively via Eqs. (27a) and (27d). As such, the HETA considering quadrature physical forcing is formulated as

$\mathbf{E}_{D}^{\dagger}+\tilde{\mathbf{E}}_{F}^{\dagger} \tilde{\mathbf{F}}+\mathbf{E}_{N}^{\dagger} \hat{\boldsymbol{\phi}}=\mathbf{0}$

which contains $R_{\mathcal{H}}$ equations with $R_{\mathcal{H}}+R_{\mathcal{F}}$ unknowns, i.e. $R_{\mathcal{H}}$ phase-shifts and $R_{\mathcal{F}}$ forcing amplitudes. To obtain a determined (solvable) problem, extra $R_{\mathcal{F}}$ constraints are needed, which can be introduced by considering the quadrature conditions, leading to phase-shift constraints, similar to the discussions in $§ 3.3$.

Firstly, consider the case where a single excitation force, at a frequency $j \omega$, is applied at a physical location denoted $\ell$. The displacement at this location is written $y_{\ell}$ and may be expressed as a sum of the modal displacements at that point, i.e.

$y_{\ell}=\sum_{i=1}^{N} \theta_{\ell, i} q_{i}$. 
Considering the modal displacement as a sum of harmonic components, as in Eq. (15), gives

$y_{\ell}=\sum_{i=1}^{N} \sum_{j \in \mathcal{H}_{i}} \theta_{\ell, i} u_{i, j}$

and hence the $j^{\text {th }}$ harmonic of the physical displacement $y_{\ell}$ may be written

$y_{\ell, j}=\sum_{i=1}^{N} \theta_{\ell, i} u_{i, j}$.

Here as $y_{\ell, j}$ represents the harmonic displacement which is in quadrature with the forcing, it may be written as a sinusoid with amplitude $Y_{\ell, j}$ and phase $\tilde{\psi}_{\ell, j}$, i.e.

$Y_{\ell, j} \cos \left(j \omega t+\tilde{\psi}_{\ell, j}\right)=\sum_{i=1}^{N} \theta_{\ell, i} U_{i, j} \cos \left(j \omega t+\phi_{i, j}\right)$,

where Eq. (17) has been used to express $u_{i, j}$ as a sinusoid and where $\phi_{i, j}=\bar{\phi}_{i, j}+\hat{\phi}_{i, j}$.

If the $j^{\text {th }}$ harmonic of the force is in quadrature with the excitation point, i.e. with $y_{\ell, j}$, then the phase, $\tilde{\psi}_{\ell, j}$, of $y_{\ell, j}$ must be equal for both the NNM and the forced case. To find how this leads to a constraint between the modal phase-shift terms, the time- and phasedependent components of Eq. (66) may be separated by first writing

$$
\begin{array}{r}
Y_{\ell, j}\left[\cos (j \omega t) \cos \left(\tilde{\psi}_{\ell, j}\right)-\sin (j \omega t) \sin \left(\tilde{\psi}_{\ell, j}\right)\right]=(67) \\
\sum_{i=1}^{N} \theta_{\ell, i} U_{i, j}\left[\cos (j \omega t) \cos \left(\phi_{i, j}\right)-\sin (j \omega t) \sin \left(\phi_{i, j}\right)\right] .
\end{array}
$$

From this, the $\cos (j \omega t)$ and $\sin (j \omega t)$ components may be balanced to give the relationships

$$
\begin{aligned}
& Y_{\ell, j} \cos \left(\tilde{\psi}_{\ell, j}\right)=\sum_{i=1}^{N} \theta_{\ell, i} U_{i, j} \cos \left(\phi_{i, j}\right) \\
& Y_{\ell, j} \sin \left(\tilde{\psi}_{\ell, j}\right)=\sum_{i=1}^{N} \theta_{\ell, i} U_{i, j} \sin \left(\phi_{i, j}\right) .
\end{aligned}
$$

The displacement amplitude $Y_{\ell, j}$ may now be removed by dividing Eq. (68b) by Eq. (68a) to give

$$
\tan \left(\tilde{\psi}_{\ell, j}\right)=\frac{\sum_{i=1}^{N} \theta_{\ell, i} U_{i, j} \sin \left(\phi_{i, j}\right)}{\sum_{i=1}^{N} \theta_{\ell, i} U_{i, j} \cos \left(\phi_{i, j}\right)} .
$$

If the phase, $\tilde{\psi}_{\ell, j}$, is equal for both the NNM and forced case, it therefore follows that Eq. (69) may be satisfied for both $\phi_{i, j}=\bar{\phi}_{i, j}+\hat{\phi}_{i, j}$ (i.e. forced responses) and $\phi_{i, j}=\bar{\phi}_{i, j}$ (i.e. NNMs). Assuming the phase-shifts are small, this may be approximated to

$$
\begin{gathered}
\frac{\left[\sum_{i=1}^{N} \theta_{\ell, i} U_{i, j} \sin \left(\bar{\phi}_{i, j}\right)\right]+\left[\sum_{i=1}^{N} \theta_{\ell, i} U_{i, j} \cos \left(\bar{\phi}_{i, j}\right) \hat{\phi}_{i, j}\right]}{\left[\sum_{i=1}^{N} \theta_{\ell, i} U_{i, j} \cos \left(\bar{\phi}_{i, j}\right)\right]-\left[\sum_{i=1}^{N} \theta_{\ell, i} U_{i, j} \sin \left(\bar{\phi}_{i, j}\right) \hat{\phi}_{i, j}\right]} \\
=\frac{\sum_{i=1}^{N} \theta_{\ell, i} U_{i, j} \sin \left(\bar{\phi}_{i, j}\right)}{\sum_{i=1}^{N} \theta_{\ell, i} U_{i, j} \cos \left(\bar{\phi}_{i, j}\right)} .
\end{gathered}
$$

This restriction leads to a constraint between the phaseshifts of the modal harmonics, and may be simplied to

$\sum_{i=1}^{N} \sum_{n=1}^{N} \theta_{\ell, i} \theta_{\ell, n} U_{i, j} U_{n, j} \cos \left(\bar{\phi}_{i, j}-\bar{\phi}_{n, j}\right) \hat{\phi}_{i, j}=0$.

In this case, each harmonic of each forcing location will be associated with an unknown forcing amplitude, but will also lead to an additional constraint given by Eq. (71). For the case where multiple forces are applied, such phase constraints introduce as many constraints as the number of unknown forcing amplitudes, $R_{\mathcal{F}}$, which may be expressed

$\mathbf{C}_{\phi} \hat{\boldsymbol{\phi}}=\mathbf{0}$,

where $\mathbf{C}_{\phi}$ denotes the phase constraint matrix, similar to that in Eq. (31). Note that, in the previous case, where quadrature harmonic forcing is considered (in $\S 3)$, the phase constraint matrix restricts zero phaseshifts to the enforced harmonics; however, here, the phase constraint matrix enforces relationships between harmonic phase-shifts.

Therefore, the number of unknowns $\left(R_{\mathcal{H}}\right.$ phaseshifts and $R_{\mathcal{F}}$ forcing amplitudes) matches the number of equations (given by the energy balancing, i.e. Eq. (62), and the phase constraints, i.e. Eq. (72)). Combining Eqs. (62) and (72), the energy balancing analysis, for quadrature physical forcing cases, may be conducted in the same form as Eq. (34), i.e.

$\mathbf{v}=\mathbf{A}^{-1} \mathbf{B}$.

where

$\mathbf{A}=\left[\begin{array}{cc}\mathbf{E}_{N}^{\dagger} & \tilde{\mathbf{E}}_{F}^{\dagger} \\ \mathbf{C}_{\phi} & \mathbf{0}\end{array}\right], \quad \mathbf{B}=\left(\begin{array}{c}-\mathbf{E}_{D}^{\dagger} \\ \mathbf{0}\end{array}\right), \quad \mathbf{v}=\left(\begin{array}{c}\hat{\boldsymbol{\phi}} \\ \tilde{\mathbf{F}}\end{array}\right)$.

This allows the phase-shifts of all harmonics, $\hat{\boldsymbol{\phi}}$, and the physical forcing amplitudes, $\tilde{\mathbf{F}}$, to be computed via known parameters obtained from NNM solutions. 


\subsection{Example 2: a quadrature physical forcing case}

To formulate the HETA for a quadrature physical forcing case, the two-mode beam model, schematically shown in Fig. 2, is again considered. Here a twoharmonic physical force is considered, given by

$f(t)=F_{1} \cos (\Omega t)+F_{2} \cos (3 \Omega t)$,

which is applied to the part-span position at $L_{1}=0.1 L$, such that the equations of motion are written

$\ddot{q}_{1}+d_{1} \dot{q}_{1}+\omega_{n 1}^{2} q_{1}+N_{q 1}\left(q_{1}, q_{2}\right)=\theta_{\ell, 1} f(t)$,

$\ddot{q}_{2}+d_{2} \dot{q}_{2}+\omega_{n 2}^{2} q_{2}+N_{q 2}\left(q_{1}, q_{2}\right)=\theta_{\ell, 2} f(t)$,

where the nonlinear forces are given by Eq. (9). The parameters of the system are the same as those considered in $\S 2.3$ and $\S 3.4$, given in Table 1 . Each modal displacement, $q_{i}$, is approximated by three harmonics, namely the odd-numbered harmonics up to the $5^{\text {th }}$ order, i.e.

$\mathcal{H}_{1}=\{1,3,5\}, \quad \mathcal{H}_{2}=\{1,3,5\}$,

such that

$q_{1} \approx u_{1,1}+u_{1,3}+u_{1,5}, \quad q_{2} \approx u_{2,1}+u_{2,3}+u_{2,5}$.

As with the example demonsrated in $§ 3.4$, the known energy-transfer coefficients are assembled into vector or matrix forms. Using Eqs. (19a), (25a) and (27a), the vector of damping energy-transfer terms, $E_{D i, j}$, is given by

$\mathbf{E}_{D}^{\dagger}=\left(\begin{array}{c}E_{D 1,1}^{\dagger} \\ E_{D 1,3}^{\dagger} \\ E_{D 1,5}^{\dagger} \\ E_{D 2,1}^{\dagger} \\ E_{D 2,3}^{\dagger} \\ E_{D 2,5}^{\dagger}\end{array}\right)=\left(\begin{array}{c}\pi \omega d_{1} U_{1,1}^{2} \\ 9 \pi \omega d_{1} U_{1,3}^{2} \\ 25 \pi \omega d_{1} U_{1,5}^{2} \\ \pi \omega d_{2} U_{2,1}^{2} \\ 9 \pi \omega d_{2} U_{2,3}^{2} \\ 25 \pi \omega d_{2} U_{2,5}^{2}\end{array}\right)$

The diagonal matrix of forcing energy coefficients, whose leading elements, $E_{P \ell, i, j}^{\dagger}$, are defined by Eq. (53), is given by

$\mathbf{E}_{P \ell}^{\dagger}=\left[\begin{array}{cccccc}E_{P \ell, 1,1}^{\dagger} & 0 & 0 & 0 & 0 & 0 \\ 0 & E_{P \ell, 1,3}^{\dagger} & 0 & 0 & 0 & 0 \\ 0 & 0 & E_{P \ell, 1,5}^{\dagger} & 0 & 0 & 0 \\ 0 & 0 & 0 & E_{P \ell, 2,1}^{\dagger} & 0 & 0 \\ 0 & 0 & 0 & 0 & E_{P \ell, 2,3}^{\dagger} & 0 \\ 0 & 0 & 0 & 0 & 0 & E_{P \ell, 2,5}^{\dagger}\end{array}\right]$,

The force reduction matrix, $\mathbf{C}_{P \ell}$, the modeshape coefficient matrix, $\boldsymbol{\theta}_{\ell}$, and the non-zero forcing amplitude vector, $\tilde{\mathbf{F}}$, may be obtained via equations from Eq. (53) to Eq. (58). These are given by

$\mathbf{C}_{P \ell}=\left[\begin{array}{llll}1 & 0 & 0 & 0 \\ 0 & 1 & 0 & 0 \\ 0 & 0 & 0 & 0 \\ 0 & 0 & 1 & 0 \\ 0 & 0 & 0 & 1 \\ 0 & 0 & 0 & 0\end{array}\right], \boldsymbol{\theta}_{\ell}=\left[\begin{array}{cc}\theta_{\ell, 1} & 0 \\ 0 & \theta_{\ell, 1} \\ \theta_{\ell, 2} & 0 \\ 0 & \theta_{\ell, 2}\end{array}\right], \tilde{\mathbf{F}}_{\ell}=\left(\begin{array}{l}F_{1} \\ F_{2}\end{array}\right)$
Using Eq. (59), the matrix of forcing energy-transfer coefficients, $\tilde{\mathbf{E}}_{F}^{\dagger}$, is written

$\tilde{\mathbf{E}}_{F \ell}^{\dagger}=\mathbf{E}_{P \ell}^{\dagger} \mathbf{C}_{P \ell} \boldsymbol{\theta}_{\ell}=\left[\begin{array}{cc}\theta_{1} E_{P 1,1}^{\dagger} & 0 \\ 0 & \theta_{1} E_{P 1,3}^{\dagger} \\ 0 & 0 \\ \theta_{2} E_{P 2,1}^{\dagger} & 0 \\ 0 & \theta_{2} E_{P 2,3}^{\dagger} \\ 0 & 0\end{array}\right]$

Next, as in $\S 3$, the matrix of nonlinear coefficients may be obtained using Eqs. (23c), (25d) and (27d), i.e.

$$
\mathbf{E}_{N}^{\dagger}=\left[\begin{array}{llllll}
E_{N 1,1}^{\dagger(1,1)} & E_{N 1,1}^{\dagger(1,3)} & E_{N 1,1}^{\dagger(1,5)} & E_{N 1,1}^{\dagger(2,1)} & E_{N 1,1}^{\dagger(2,3)} & E_{N 1,1}^{\dagger(2,5)} \\
E_{N 1,3}^{\dagger(1,1)} & E_{N 1,3}^{\dagger(1,3)} & E_{N 1,3}^{\dagger(1,5)} & E_{N 1,3}^{\dagger(2,1)} & E_{N 1,3}^{\dagger(2,3)} & E_{N 1,3}^{\dagger(2,5)} \\
E_{N 1,5}^{\dagger(1,1)} & E_{N 1,5}^{\dagger(1,3)} & E_{N 1,5}^{\dagger(1,5)} & E_{N 1,5}^{\dagger(2,1)} & E_{N 1,5}^{\dagger(2,3)} & E_{N 1,5}^{\dagger(2,5)} \\
E_{N 2,1}^{\dagger(1,1)} & E_{N 2,1}^{\dagger(1,3)} & E_{N 2,1}^{\dagger(1,5)} & E_{N 2,1}^{\dagger(2,1)} & E_{N 2,1}^{\dagger(2,3)} & E_{N 2,1}^{\dagger(2,5)} \\
E_{N 2,3}^{\dagger(1,1)} & E_{N 2,3}^{\dagger(1,3)} & E_{N 2,3}^{\dagger(1,5)} & E_{N 2,3}^{\dagger(2,1)} & E_{N 2,3}^{\dagger(2,3)} & E_{N 2,3}^{\dagger(2,5)} \\
E_{N 2,5}^{\dagger(1,1)} & E_{N 2,5}^{\dagger(1,3)} & E_{N 2,5}^{\dagger(1,5)} & E_{N 2,5}^{\dagger(2,1)} & E_{N 2,5}^{\dagger(2,3)} & E_{N 2,5}^{\dagger(2,5)}
\end{array}\right],
$$

where the elements in this matrix may be computed using Eq. (19c).

The phase-shift constraints, defined in Eq. (71), may be assembled into matrix form as Eq. (72), where the matrix of phase-shift constraint coefficients, $\mathbf{C}_{\phi}$, and vector of phase-shifts, $\hat{\phi}$, are given

$$
\begin{aligned}
\mathbf{C}_{\phi} & =\left[\begin{array}{cccccc}
C_{\phi 1,1} & 0 & 0 & C_{\phi 2,1} & 0 & 0 \\
0 & C_{\phi 1,3} & 0 & 0 & C_{\phi 2,3} & 0
\end{array}\right], \\
\hat{\boldsymbol{\phi}} & =\left(\begin{array}{llllll}
\hat{\phi}_{11} & \hat{\phi}_{13} & \hat{\phi}_{15} & \hat{\phi}_{21} & \hat{\phi}_{23} & \hat{\phi}_{25}
\end{array}\right)^{\top},
\end{aligned}
$$

where

$$
\begin{aligned}
& C_{\phi 1,1}=\theta_{\ell, 1}^{2} U_{1,1}^{2}+\theta_{\ell, 1} \theta_{\ell, 2} U_{1,1} U_{2,1} \cos \left(\bar{\phi}_{1,1}-\bar{\phi}_{2,1}\right), \\
& C_{\phi 2,1}=\theta_{\ell, 2}^{2} U_{2,1}^{2}+\theta_{\ell, 2} \theta_{\ell, 1} U_{2,1} U_{1,1} \cos \left(\bar{\phi}_{2,1}-\bar{\phi}_{1,1}\right), \\
& C_{\phi 1,3}=\theta_{\ell, 1}^{2} U_{1,3}^{2}+\theta_{\ell, 1} \theta_{\ell, 2} U_{1,3} U_{2,3} \cos \left(\bar{\phi}_{1,3}-\bar{\phi}_{2,3}\right), \\
& C_{\phi 2,3}=\theta_{\ell, 2}^{2} U_{2,3}^{2}+\theta_{\ell, 2} \theta_{\ell, 1} U_{2,3} U_{1,3} \cos \left(\bar{\phi}_{2,3}-\bar{\phi}_{1,3}\right) .
\end{aligned}
$$

Combining equations from Eq. (79) to Eq. (84), the energy transfer analysis may be formulated in the form as Eq. (73). Therefore, the unknowns, i.e. the physical forcing amplitudes, $\tilde{\mathbf{F}}_{\ell}$, and the phase-shifts, $\hat{\boldsymbol{\phi}}$, may be computed. Here the results obtained from HETA are compared with the forced responses obtained via the force appropriation technique, proposed in [23].

Figure 8 shows the solved parameters, i.e. physical forcing amplitudes, $\left|F_{1}\right|$ and $\left|F_{2}\right|$, and harmonic phaseshifts, i.e. $\hat{\phi}_{1,1}, \hat{\phi}_{1,3}, \hat{\phi}_{1,5}, \hat{\phi}_{2,1}, \hat{\phi}_{2,3}$ and $\hat{\phi}_{2,5}$. In all panels of Fig. 8, the predicted results obtained via HETA are shown as blue solid lines whilst the simulated results obtained using force appropriation are shown as red lines with dots. The forcing amplitudes obtained from both techniques are indistinguishable - see panels $(a)$ and $(e)$ in Fig. 8. Excellent agreement is generally 

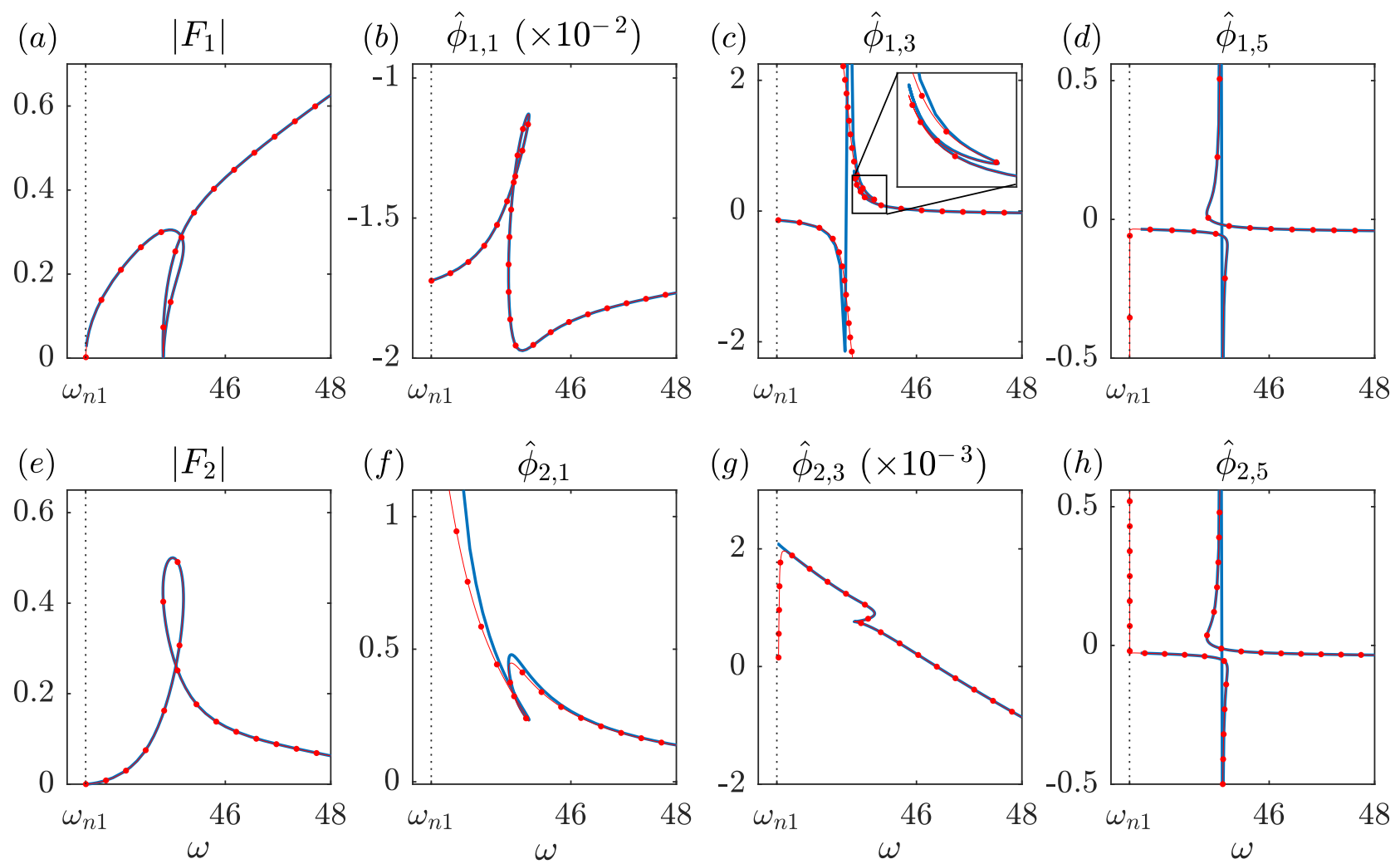

Fig. 8 A comparison between the predictions of HETA (using a three-harmonic approximation for each mode) and the numerically-simulated forced responses. Panels $(a)$ and $(e)$ show the frequency, $\omega$, against the forcing amplitudes $F_{1}$ and $F_{2}$ respectively. Panels $(b),(c),(d),(f),(g)$ and $(h)$ show the frequency, $\omega$, against the phase-shift parameters, $\hat{\phi}_{1,1}, \hat{\phi}_{1,3}, \hat{\phi}_{1,5}$, $\hat{\phi}_{2,1}, \hat{\phi}_{2,3}$ and $\hat{\phi}_{2,5}$ respectively. In all panels, the blue lines represent analytically-predicted values (using the NNM data), the red lines (with dots) represent the numerical results and the dotted-black line denotes the first linear natural frequency..

achieved between analytical predictions and numerical simulations for most phase-shifts. One discrepency lies in the predictions of $\hat{\phi}_{1,3}$ near the region where a strong internal resonance is shown between two linear modes (the loop region in panels $(a)$ and $(e)$ ). This discrepancy may arise from the large phase-shift in this region, whilst this technique is derived based on a small phaseshift assumption. Nonetheless, the numerical results indicate a drastic change in the sign of phase-shift from positive to negative or vice versa, which is also captured by the results obtained from HETA. The other discrepancy lies in the prediction of $\hat{\phi}_{2,1}$ near the natural frequency region, however, the trend over response frequency is again captured in good agreement.

\subsection{Example 3: predicting isolas via HETA}

Determining the existence of the isolated forced responses, or isolas, is of great importance in analysis of nonlinear responses $[19,9,5]$. Using the energy balancing analysis to predict the existence of isolas has proven to be an efficient method [9]. However, as discussed in Fig. 3(b), when responses contain multiple significant harmonics, predicting these isolas can be challenging - a key motivation for this work. In this section, an improved and more robust method is demonstrated in predicting the existence of isolas using the energy balancing analysis proposed in this paper.

For comparison, the example system, considered in $\S 2$, is revisited. The nonlinear beam is modelled by a two-mode model, described by Eqs. (8) and (9), where the parameters are given in Table 1. A single physical forcing, expressed by Eq. (7), is applied to the part-span position at $L_{1}=0.2 L$ (the same excitation scenario as that shown in Fig. 3(b)). Here, each mode is approximated by three harmonics, i.e. the odd-numbered harmonics up to $5^{\text {th }}$ order, as considered in $\S 4.2$. As such, the formulation of META is equivalent to that given in $\S 4.2$ with $F_{2}=0$, and hence it is not re-formulated here.

The solved parameters, i.e. forcing amplitude, $F_{1}$, and phase-shifts, $\hat{\phi}_{1,1}, \hat{\phi}_{1,3}, \hat{\phi}_{1,5}, \hat{\phi}_{2,1}, \hat{\phi}_{2,3}$ and $\hat{\phi}_{2,5}$, are shown as blue lines in Fig. 9. In panel $(a)$, the forcing amplitudes, solved via the single-harmonic META, are shown as a green line in comparison with that obtained via the multi-harmonic HETA. Near the natu- 

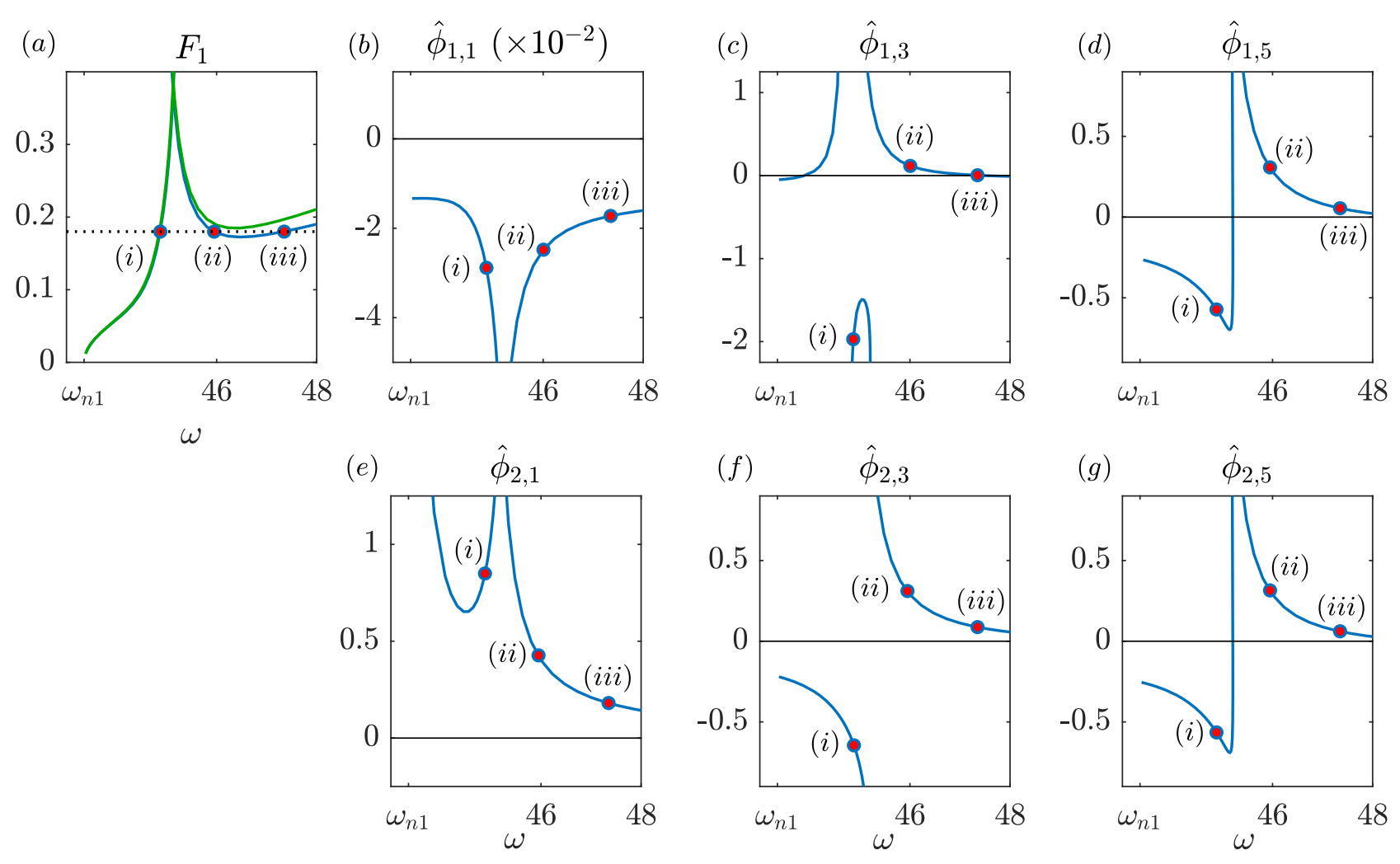

Fig. 9 Predicting the existence of an isola using Harmonic-level Energy Transfer Analysis (HETA). The HETA-predicted forcing amplitude and phase-shifts are shown as blue lines in each panel; the META-predicted forcing amplitude is shown as a green line for comparison in panel $(a)$. Considering a forcing amplitude of $F_{1}=0.18$, denoted by a dotted line in panel $(a)$, the resonant crossing points on the NNM branch are labelled by solid dots and marked by (i), (ii) and (iii).

ral frequency, the difference between results obtained via META and HETA are indistinguishable; as response frequency increases, the discrepancy between the two lines grows, indicating an increasing significance of the harmonics. Considering a forcing amplitude of $F_{1}=0.18$, HETA predicts one intersection, also shown in Fig. 3(b) (the existence of an isola is not captured); whilst META predicts three intersections, labelled by solid dots and marked by $(i),(i i)$ and (iii) in each panel of Fig. 9. This denotes that there are three resonant crossing points between the NNM branch and the forced responses. The perturbations, from NNM solutions to resonances, for these three predictions can be evaluated by referring to the phase-shifts from panels (b) to $(g)$. In each panel, it is shown that the phaseshift at point $(i)$ is the largest of the three, whilst that at point (iii) is the smallest. Thus, it can be expected that the largest perturbation, from an NNM solution to a resonance, occurs at point $(i)$, and the smallest one is at point $(i i i)$.

To verify these results, the forced responses of the beam system are computed via numerical continuation and shown as red lines in the left panel of Fig. 10. Three HETA-predicted crossing points on the NNM branch are likewise denoted by solid dots, whilst the numerically obtained resonances are labelled by hollow dots. The corresponding time-parameterised responses for the NNM solutions, HETA-predicted resonances (perturbing the NNM solutions with predicted phase-shifts) and the numerically obtained resonances are shown in the right-hand panels. Shown in the left panel, the existence of three resonances are well predicted using HETA, capturing the resonances on both the primary response curve and the isola. In the right panels, the perturbations from NNM solutions to resonances are accurately captured by the phase-shifts HETA-predicted responses well match the numerically computed resonances. In addition, the levels of perturbations are well captured by the phase-shifts - the largest perturbation occurs at point $(i)$ and the smallest one at $($ iii $)$.

In this example, predicting the existence of an isola is considered via HETA. Results show that the HETA can accurately predict all the resonant crossing points between the NNM branch and forced responses, showing an improved accuracy when compared with META. The perturbations from crossing points on the NNM 

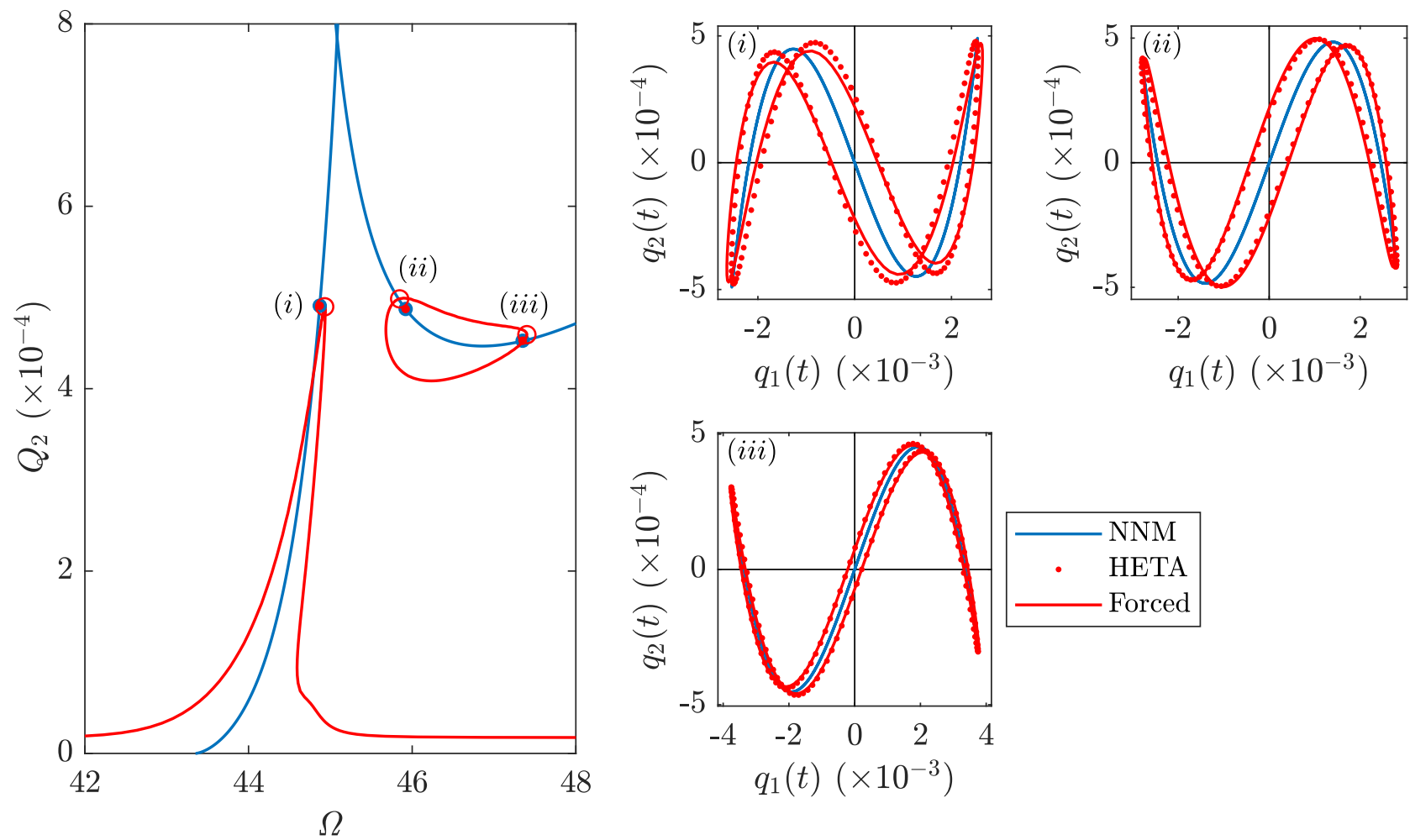

Fig. 10 Verification and comparison of the HETA-predicted results using forced responses. In the left panel, the forced responses of the beam system with $F_{1}=0.18$ is computed via numerical continuation and shown as red lines. The HETApredicted crossing points on the NNM branch are shown as solid dots, denoted by $(i)$, (ii) and (iii); whilst the numerically obtained resonances are labelled by hollow dots. In the right panels, the time-parameterised responses for crossing points on the NNM branch, the HETA-predicted resonances and the numerically obtained resonances are compared.

branch to the resonances are also shown to be accurately captured by the phase-shifts.

\section{Conclusions}

In this paper, we have considered energy balancing across multiple harmonics of nonlinear normal modes (NNMs) for predicting resonances.

The energy balancing mechanism is established by accounting for the net energy transfer, arising from nonlinearity, external forcing, and damping, between harmonics of an NNM over a period of response. With known parameter inputs from NNMs, combined with force reduction and restrictions on harmonic phaseshifts, a semi-analytical framework has been developed for accurate and efficient predictions of resonances. Two excitation scenarios, namely where the excitations are in quadrature with 1) harmonic displacements and 2) physical displacements, have been considered to cover practical applications.

We have then considered three specific application cases to demonstrate the applicability of this technique. For all these cases, the proposed computationally cheap technique showed excellent agreement with the computationally expensive numerical simulations. In the third example, the technique was used to predict the existence of an isola that contains multiple significant harmonics. The locations of resonances and the deviations from NNMs to resonances were both accurately predicted.

Owing to the semi-analytical framework, this technique can be easily extended to account for more complex scenarios, e.g. multiple harmonics of multiple modes of NNMs, and multiple forcing harmonics at multiple locations, without adding much computational effort.

\section{Data accessibility}

This article has no additional data.

\section{Conflict of interest}

The authors declare that they have no conflict of interest. 
Acknowledgements The authors gratefully acknowledge the support of the Engineering and Physical Sciences Research Council (EPSRC) and the China Scholarship Council (CSC): S.A.N. is supported by the EPSRC (grant no. $\mathrm{EP} / \mathrm{R} 006768 / 1)$ and D.H is supported by a scholarship from the CSC.

\section{A Computing the energy-loss terms}

Equation (13) shows the relationship between the damping, forcing and nonlinear energy-loss terms for the $j^{\text {th }}$ harmonic of the $i^{\text {th }}$ mode, where these terms are computed using Eq. (12). It is now shown that these terms may be written as linear functions of the forcing amplitudes, $P_{i, j}$, and phaseshifts, $\hat{\phi}_{i, j}$, which are assumed to be unknown.

\section{A.1 Damping energy-loss term}

Substituting the harmonic expansion of the modal velocities, from Eq. (17), into the expression for the damping energy loss, Eq. (12a), gives

$E_{D i, j}=\int_{0}^{T}\left[d_{i} \sum_{k \in \mathcal{H}_{i}} \dot{u}_{i, k}\right] \dot{u}_{i, j} \mathrm{~d} t$.

Noting that $\dot{u}_{i, 0}=0$ and that

$\int_{0}^{T} \dot{u}_{i, k} \dot{u}_{i, j} \mathrm{~d} t=0, \quad$ when: $k \neq j$,

Eq. (86) may be simplified to

$E_{D i, j}=d_{i} \int_{0}^{T} \dot{u}_{i, j}^{2} \mathrm{~d} t$

Substituting the expression for $\dot{u}_{i, j}$, from Eq. (17), into Eq. (88) then gives

$$
\begin{aligned}
E_{D i, j} & =d_{i} \int_{0}^{T}\left[-j \omega U_{i, j} \sin \left(j \omega t+\bar{\phi}_{i, j}+\hat{\phi}_{i, j}\right)\right]^{2} \mathrm{~d} t \\
& =\pi j^{2} \omega d_{i} U_{i, j}^{2}
\end{aligned}
$$

where $T=2 \pi \omega^{-1}$ has been used.

\section{A.2 Forcing energy-loss term}

Substituting the harmonic expansion of external forcing, Eq. (16), into the expression for the forcing energy loss, Eq. (12b), gives

$E_{P i, j}=-\int_{0}^{T}\left[\sum_{k \in \mathcal{H}_{i}} p_{i, k}\right] \dot{u}_{i, j} \mathrm{~d} t$.

Noting that

$$
\begin{aligned}
& \int_{0}^{T} p_{i, k} \dot{u}_{i, j} \mathrm{~d} t=0, \quad \text { when: } k \neq j, \\
& \text { and: } \quad \int_{0}^{T} p_{i, 0} \dot{u}_{i, j} \mathrm{~d} t=0, \quad \text { for all } j,
\end{aligned}
$$

Eq. (90) is simplified to

$$
E_{P i, j}=-\int_{0}^{T} p_{i, j} \dot{u}_{i, j} \mathrm{~d} t
$$

The harmonic forcing, $p_{i, j}$ and velocity, $\dot{u}_{i, j}$, are assumed as

$$
\begin{aligned}
p_{i, j} & =-P_{i, j} \sin \left(j \omega t+\psi_{i, j}\right), \\
\dot{u}_{i, j} & =-j \omega U_{i, j} \sin \left(j \omega t+\phi_{i, j}\right) \\
& =-j \omega U_{i, j} \sin \left(j \omega t+\bar{\phi}_{i, j}+\hat{\phi}_{i, j}\right) .
\end{aligned}
$$

where $P_{i, j}$ and $\psi_{i, j}$ are the amplitude and phase of the forcing respectively. Substituting the expressions for $p_{i, j}$ and $\dot{u}_{i, j}$ into Eq. (92), gives

$$
\begin{aligned}
& E_{P i, j}= \int_{0}^{T}\left[-P_{i, j} \sin \left(j \omega t+\psi_{i, j}\right)\right] \times \\
& {\left[j \omega U_{i, j} \sin \left(j \omega t+\bar{\phi}_{i, j}+\hat{\phi}_{i, j}\right)\right] \mathrm{d} t } \\
& \approx-\pi j P_{i, j} U_{i, j} \cos \left(\psi_{i, j}-\bar{\phi}_{i, j}\right)
\end{aligned}
$$

where it has been assumed that the phase-shift, $\hat{\phi}_{i, j}$, is small. The phase difference, $\psi_{i, j}-\bar{\phi}_{i, j}$, accounts for the effect of external forcing on harmonic $u_{i, j}$ - either an energy gain or an energy loss.

For the case where quadrature harmonic forcings are considered, i.e. $\S 3$, the phase of the harmonic forcing, $\psi_{i, j}$, may be assumed to be equal the phase of the velocity, $\bar{\phi}_{i, j}$. This further simplifies Eq. (94) to

$E_{P i, j} \approx-\pi j P_{i, j} U_{i, j}$.

Assuming the displacement amplitude, $U_{i, j}$, is known but the forcing amplitude, $P_{i, j}$, is unknown, the forcing energy term may be separated into the known, $E_{P i, k}^{\dagger}$, and unknown, $P_{i, k}$, components, i.e.

$E_{P i, j} \approx P_{i, j} E_{P i, j}^{\dagger}, \quad$ where: $\quad E_{P i, j}^{\dagger}=-\pi j U_{i, j}$,

where the dagger, $\bullet^{\dagger}$, denotes a known term.

Whilst, for the case where quadrature physical forcings are considered, as in $\S 4$, the phase relationship between the external forcing and the harmonic can be determined using the phase constraints Eq. (69) with $\phi_{i, j}=\bar{\phi}_{i, j}$, i.e.

$\tan \left(\psi_{\ell, j}\right)=\frac{\sum_{i=1}^{N} \theta_{\ell, i} U_{i, j} \sin \left(\bar{\phi}_{i, j}\right)}{\sum_{i=1}^{N} \theta_{\ell, i} U_{i, j} \cos \left(\bar{\phi}_{i, j}\right)}$.

In this context, the phase difference, $\psi_{i, j}-\bar{\phi}_{i, j}$, can be evaluated using the NNM solutions and thus, it can be seen as a known parameter.

\section{A.3 Nonlinear energy-loss term}

As with the forcing energy-loss term, the known and unknown components must be separated in the nonlinear energy-loss term, Eq. (12c). Before considering the (unknown) phaseshifts, $\hat{\phi}_{i, j}$, the responses are divided into the NNM responses (assumed to be known) and the perturbations (assumed to be unknown) - i.e. $q_{i}=\bar{q}_{i}+\hat{q}_{i}$ where, as previously, $\bar{q}_{i}$ represents the NNM response and where $\hat{q}_{i}$ is the perturbation to the $i^{\text {th }}$ modal displacement due to the forcing. Following this, the vector of modal displacements may be written $\mathbf{q}=\overline{\mathbf{q}}+\hat{\mathbf{q}}$, and the harmonics are written $u_{i, j}=\bar{u}_{i, j}+\hat{u}_{i, j}$. Substituting these into the nonlinear energy-loss term, Eq. (12c), leads to

$E_{N i, j}=\int_{0}^{T}\left[N_{q i}(\overline{\mathbf{q}}+\hat{\mathbf{q}})\right]\left[\dot{\bar{u}}_{i, j}+\dot{\hat{u}}_{i, j}\right] \mathrm{d} t$. 
Assuming the perturbation terms are small, the Taylor series expansion may be applied to the nonlinear force to give

$$
\begin{aligned}
& E_{N i, j} \approx \int_{0}^{T}\left[N_{q i}(\overline{\mathbf{q}})+\left(\sum_{n=1}^{N}\left[\frac{\mathrm{d} N_{q i}}{\mathrm{~d} q_{n}}\right]_{\mathbf{q}=\overline{\mathbf{q}}} \hat{q}_{n}\right)\right] \times \\
& {\left[\dot{\bar{u}}_{i, j}+\dot{\hat{u}}_{i, j}\right] \mathrm{d} t \text {, }}
\end{aligned}
$$

which may be expanded out to

$$
\begin{aligned}
E_{N i, j} \approx \int_{0}^{T} & {\left[N_{q i}(\overline{\mathbf{q}}) \dot{\bar{u}}_{i, j}\right]+\left[N_{q i}(\overline{\mathbf{q}}) \dot{\hat{u}}_{i, j}\right] } \\
+ & {\left[\left(\sum_{n=1}^{N}\left[\frac{\mathrm{d} N_{q i}}{\mathrm{~d} q_{n}}\right]_{\mathbf{q}=\overline{\mathbf{q}}} \hat{q}_{n}\right) \dot{\bar{u}}_{i, j}\right] } \\
+ & {\left[\left(\sum_{n=1}^{N}\left[\frac{\mathrm{d} N_{q i}}{\mathrm{~d} q_{n}}\right]_{\mathbf{q}=\overline{\mathbf{q}}} \hat{q}_{n}\right) \dot{\hat{u}}_{i, j}\right] \mathrm{d} t . }
\end{aligned}
$$

As the unperturbed response represents an NNM, the first term in the integral of Eq. (100) represents the net energy transfer due to the nonlinear coupling of an NNM response. As an NNM cannot exhibit a net energy transfer, it follows that

$\int_{0}^{T} N_{q i}(\overline{\mathbf{q}}) \dot{\bar{u}}_{i, j} \mathrm{~d} t=0$

Assuming that the perturbations, $\hat{q}_{n}$ and $\hat{u}_{i, j}$, are small it also follows that the final term in the integral of Eq. (100) is negligible. Therefore, Eq. (100) may be further approximated to

$$
\begin{aligned}
E_{N i, j} \approx \int_{0}^{T} & {\left[N_{q i}(\overline{\mathbf{q}}) \dot{\hat{u}}_{i, j}\right] } \\
+ & {\left[\left(\sum_{n=1}^{N}\left[\frac{\mathrm{d} N_{q i}}{\mathrm{~d} q_{n}}\right]_{\mathbf{q}=\overline{\mathbf{q}}} \hat{q}_{n}\right) \dot{\bar{u}}_{i, j}\right] \mathrm{d} t . }
\end{aligned}
$$

The perturbations, $\hat{q}_{n}$ and $\hat{u}_{i, j}$, are now written in terms of the phase-shifts, $\hat{\phi}_{i, j}$. To achieve this, Eq. (17) is used to write the $j^{\text {th }}$ harmonic of the $i^{\text {th }}$ mode as

$$
\begin{aligned}
u_{i, j}=\bar{u}_{i, j}+\hat{u}_{i, j}= & U_{i, j} \cos \left(j \omega t+\bar{\phi}_{i, j}+\hat{\phi}_{i, j}\right), \\
= & U_{i, j} \cos \left(j \omega t+\bar{\phi}_{i, j}\right) \cos \left(\hat{\phi}_{i, j}\right) \\
& \quad-U_{i, j} \sin \left(j \omega t+\bar{\phi}_{i, j}\right) \sin \left(\hat{\phi}_{i, j}\right), \\
\approx & \bar{u}_{i, j}-U_{i, j} \sin \left(j \omega t+\bar{\phi}_{i, j}\right) \hat{\phi}_{i, j}, \quad(103)
\end{aligned}
$$

where it has been assumed that $\hat{\phi}_{i, j}$ is small. It therefore follows that

$\hat{u}_{i, j} \approx-U_{i, j} \sin \left(j \omega t+\bar{\phi}_{i, j}\right) \hat{\phi}_{i, j}=\frac{1}{j \omega} \dot{\bar{u}}_{i, j} \hat{\phi}_{i, j}$,

and hence the perturbation terms in Eq. (102) may be written $\dot{\hat{u}}_{i, j} \approx \frac{1}{j \omega} \ddot{\bar{u}}_{i, j} \hat{\phi}_{i, j}, \quad$ and $\quad \hat{q}_{n}=\sum_{k \in \mathcal{H}_{n}} \frac{\hat{\phi}_{n, k}}{k \omega} \dot{\bar{u}}_{n, k}$.

Substituting these into Eq. (102) gives

$$
\begin{aligned}
E_{N i, j} \approx & \int_{0}^{T}\left[\sum_{n=1}^{N} \frac{\partial N_{q i}}{\partial q_{n}}\left(\sum_{k \in \mathcal{H}_{n}} \frac{\hat{\phi}_{n, k}}{k \omega} \dot{\bar{u}}_{n, k}\right)\right] \dot{\bar{u}}_{i, j} \mathrm{~d} t \\
& +\frac{\hat{\phi}_{i, j}}{j \omega} \int_{0}^{T} N_{q i}(\overline{\mathbf{q}}) \ddot{\bar{u}}_{i, j} \mathrm{~d} t \\
\approx & {\left[\sum_{n=1}^{N} \sum_{k \in \mathcal{H}_{n}} \frac{\hat{\phi}_{n, k}}{k \omega}\left(\int_{0}^{T} \frac{\partial N_{q i}}{\partial q_{n}} \dot{\bar{u}}_{n, k} \dot{\bar{u}}_{i, j} \mathrm{~d} t\right)\right] }
\end{aligned}
$$

$$
+\frac{\hat{\phi}_{i, j}}{j \omega} \int_{0}^{T} N_{q i}(\overline{\mathbf{q}}) \ddot{\bar{u}}_{i, j} \mathrm{~d} t .
$$

As with the forcing energy-loss term, this may be separated into the known components (i.e. the NNM responses) and unknown components (the phase-shifts) as follows

$E_{N i, j} \approx \sum_{n=1}^{N} \sum_{k \in \mathcal{H}_{n}} E_{N i, j}^{\dagger(n, k)} \hat{\phi}_{n, k}$,

where the known component is written

$$
E_{N i, j}^{\dagger(n, k)}=\left\{\begin{array}{l}
\frac{1}{k \omega} \int_{0}^{T} \frac{\partial N_{q i}}{\partial q_{n}} \dot{\bar{u}}_{i, j} \dot{\bar{u}}_{n, k} \mathrm{~d} t, \\
\text { when: }\{i, j\} \neq\{n, k\}, \\
\frac{1}{j \omega} \int_{0}^{T} \frac{\partial N_{q i}}{\partial q_{i}} \dot{\bar{u}}_{i, j}^{2} \mathrm{~d} t \\
\quad+\frac{1}{j \omega} \int_{0}^{T} N_{q i} \ddot{\bar{u}}_{i, j} \mathrm{~d} t, \\
\text { when: }\{i, j\}=\{n, k\} .
\end{array}\right.
$$

As in A.2, the dagger, $\bullet^{\dagger}$, denotes a known term.

\section{References}

1. Cammarano, A., Hill, T., Neild, S., Wagg, D.: Bifurcations of backbone curves for systems of coupled nonlinear two mass oscillator. Nonlinear Dynamics 77(1), 311-320 (2014)

2. Cenedese, M., Haller, G.: How do conservative backbone curves perturb into forced responses? a melnikov function analysis. Proceedings of the Royal Society A: Mathematical, Physical and Engineering Sciences 476(2234), 20190494 (2020). DOI 10.1098/rspa.2019.0494

3. Dankowicz, H., Schilder, F.: Recipes for Continuation. Society for Industrial and Applied Mathematics, Philadelphia, PA (2013). DOI 10.1137/1.9781611972573

4. Denis, V., Jossic, M., Giraud-Audine, C., Chomette, B., Renault, A., Thomas, O.: Identification of nonlinear modes using phase-locked-loop experimental continuation and normal form. Mechanical Systems and Signal Processing 106, 430-452 (2018). DOI https://doi.org/10.1016/j.ymssp.2018.01.014

5. Detroux, T., Noël, J.P., Virgin, L.N., Kerschen, G.: Experimental study of isolas in nonlinear systems featuring modal interactions. PLOS ONE 13(3), 1-25 (2018). DOI 10.1371/journal.pone.0194452

6. Glendinning, P.: Stability, instability and chaos: an introduction to the theory of nonlinear differential equations. Cambridge university press (1994)

7. Hajjaj, A.Z., Hafiz, M.A., Younis, M.I.: Mode coupling and nonlinear resonances of mems arch resonators for bandpass filters. Scientific reports 7(1), 1-7 (2017)

8. Haller, G., Ponsioen, S.: Nonlinear normal modes and spectral submanifolds: existence, uniqueness and use in model reduction. Nonlinear Dynamics 86(3), 1493-1534 (2016). DOI 10.1007/s11071-016-2974-z

9. Hill, T., Neild, S., Cammarano, A.: An analytical approach for detecting isolated periodic solution branches in weakly nonlinear structures. Journal of Sound and Vibration 379, 150 - 165 (2016). DOI 10.1016/j.jsv.2016.05.030

10. Hill, T., Neild, S., Cammarano, A., Wagg, D.: The influence of phase-locking on internal resonance from a nonlinear normal mode perspective. Journal of Sound and Vibration 379, 135 - 149 (2016). DOI 10.1016/j.jsv.2016.05.028 
11. Hill, T.L., Cammarano, A., Neild, S.A., Barton, D.A.W.: Identifying the significance of nonlinear normal modes. Proceedings of the Royal Society A: Mathematical, Physical and Engineering Sciences 473(2199), 20160789 (2017). DOI 10.1098/rspa.2016.0789

12. Hill, T.L., Cammarano, A., Neild, S.A., Wagg, D.J.: Interpreting the forced responses of a two-degree-offreedom nonlinear oscillator using backbone curves. Journal of Sound and Vibration 349, 276-288 (2015). DOI $10.1016 /$ j.jsv.2015.03.030

13. Hong, D., Hill, T.L., Neild, S.A.: Conditions for the existence of isolated backbone curves. Proceedings of the Royal Society A: Mathematical, Physical and Engineering Sciences 475(2232), 20190374 (2019). DOI 10.1098/rspa.2019.0374

14. Hong, D., Hill, T.L., Neild, S.A.: Understanding targeted energy transfer from a symmetry breaking perspective. Proceedings of the Royal Society A 477(2251), 20210045 (2021)

15. Hong, D., Nicolaidou, E., Hill, T.L., Neild, S.A.: Identifying phase-varying periodic behaviour in conservative nonlinear systems. Proceedings of the Royal Society A 476(2237), 20200028 (2020)

16. Karaağaçlı, T., Özgüven, H.N.: Experimental modal analysis of nonlinear systems by using responsecontrolled stepped-sine testing. Mechanical Systems and Signal Processing 146, 107023 (2021). DOI https://doi.org/10.1016/j.ymssp.2020.107023

17. Kerschen, G., Peeters, M., Golinval, J.C., Stéphan, C.: Nonlinear modal analysis of a full-scale aircraft. Journal of Aircraft 50(5), 1409-1419 (2013). DOI 10.2514/1.C031918

18. Kerschen, G., Peeters, M., Golinval, J.C., Vakakis, A.F.: Nonlinear normal modes, part i: A useful framework for the structural dynamicist. Mechanical Systems and Signal Processing 23(1), 170-194 (2009). DOI 10.1016/j.ymssp.2008.04.002

19. Kuether, R., Renson, L., Detroux, T., Grappasonni, C., Kerschen, G., Allen, M.: Nonlinear normal modes, modal interactions and isolated resonance curves. Journal of Sound and Vibration 351, 299 - 310 (2015). DOI $10.1016 /$ j.jsv.2015.04.035

20. Kuether, R.J., Allen, M.S., Hollkamp, J.J.: Modal substructuring of geometrically nonlinear finite element models with interface reduction. AIAA Journal 55(5), 1695-1706 (2017). DOI 10.2514/1.J055215

21. Nayfeh, A.H., Mook, D.T., Holmes, P.: Nonlinear oscillations (1980)

22. Nicolaidou, E., Hill, T.L., Neild, S.A.: Detecting internal resonances during model reduction. Proceedings of the Royal Society A: Mathematical, Physical and Engineering Sciences 477(2250), 20210215 (2021). DOI 10.1098/rspa.2021.0215

23. Peeters, M., Kerschen, G., Golinval, J.: Dynamic testing of nonlinear vibrating structures using nonlinear normal modes. Journal of Sound and Vibration 330(3), $486-$ 509 (2011). DOI 10.1016/j.jsv.2010.08.028

24. Peeters, M., Kerschen, G., Golinval, J.: Modal testing of nonlinear vibrating structures based on nonlinear normal modes: Experimental demonstration. Mechanical Systems and Signal Processing 25(4), 1227-1247 (2011). DOI https://doi.org/10.1016/j.ymssp.2010.11.006

25. Peter, S., Leine, R.I.: Excitation power quantities in phase resonance testing of nonlinear systems with phase-locked-loop excitation. Mechanical Systems and Signal Processing 96, 139-158 (2017). DOI https://doi.org/10.1016/j.ymssp.2017.04.011
26. Renson, L., Barton, D.A.W., Neild, S.A.: Experimental tracking of limit-point bifurcations and backbone curves using control-based continuation. International Journal of Bifurcation and Chaos 27(01), 1730002 (2017). DOI 10.1142/S0218127417300026

27. Renson, L., Gonzalez-Buelga, A., Barton, D., Neild, S.: Robust identification of backbone curves using control-based continuation. Journal of Sound and Vibration 367, 145-158 (2016). DOI https://doi.org/10.1016/j.jsv.2015.12.035

28. Renson, L., Hill, T.L., Ehrhardt, D.A., Barton, D.A.W., Neild, S.A.: Force appropriation of nonlinear structures. Proceedings of the Royal Society A: Mathematical, Physical and Engineering Sciences 474(2214), 20170880 (2018). DOI 10.1098/rspa.2017.0880

29. Rosenberg, R.M.: Normal Modes of Nonlinear DualMode Systems. Journal of Applied Mechanics 27(2), 263268 (1960). DOI 10.1115/1.3643948

30. Rosenberg, R.M.: The Normal Modes of Nonlinear nDegree-of-Freedom Systems. Journal of Applied Mechanics 29(1), 7-14 (1962). DOI 10.1115/1.3636501. URL https://hal.archives-ouvertes.fr/hal-01344457

31. Scheel, M.: Nonlinear modal testing of damped structures: Velocity feedback vs. phase resonance. Mechanical Systems and Signal Processing 165, 108305 (2022). DOI https://doi.org/10.1016/j.ymssp.2021.108305

32. Shaw, A., Hill, T., Neild, S., Friswell, M.: Periodic responses of a structure with 3:1 internal resonance. Mechanical Systems and Signal Processing 81, 19 - 34 (2016). DOI 10.1016/j.ymssp.2016.03.008

33. Shaw, S.W., Pierre, C.: Normal modes for non-linear vibratory systems. Journal of sound and vibration 164(1), 85-124 (1993)

34. Shearer, C.M., Cesnik, C.E.S.: Nonlinear flight dynamics of very flexible aircraft. Journal of Aircraft 44(5), 1528-1545 (2007). DOI 10.2514/1.27606. URL https://doi.org/10.2514/1.27606

35. Sun, Y., Vizzaccaro, A., Yuan, J., Salles, L.: An extended energy balance method for resonance prediction in forced response of systems with non-conservative nonlinearities using damped nonlinear normal mode. Nonlinear Dynamics 103(4), 3315-3333 (2021)

36. Sun, Y., Yuan, J., Vizzaccaro, A., Salles, L.: Comparison of different methodologies for the computation of damped nonlinear normal modes and resonance prediction of systems with non-conservative nonlinearities. Nonlinear Dynamics pp. 1-31 (2021)

37. Touzé, C., Amabili, M.: Nonlinear normal modes for damped geometrically nonlinear systems: Application to reduced-order modelling of harmonically forced structures. Journal of Sound and Vibration 298(4), 958-981 (2006). DOI https://doi.org/10.1016/j.jsv.2006.06.032

38. Touzé, C.: Normal form theory and nonlinear normal modes: theoretical settings and applications. In: Modal analysis of nonlinear mechanical systems, pp. 75-160. Springer (2014)

39. Volvert, M., Kerschen, G.: Phase resonance nonlinear modes of mechanical systems. Journal of Sound and Vibration 511, 116355 (2021). DOI https://doi.org/10.1016/j.jsv.2021.116355

40. Wagg, D., Neild, S.: Nonlinear vibration with control. Springer International Publishing Switzerland (2015) 